\title{
Uniqueness of the Physical Vacuum and the Wightman Functions in the Infinite Volume Limit for Some non Polynomial Interactions
}

\author{
Sergio Albeverio and Raphael Høegh-Krohn \\ Institute of Mathematics, University of Oslo, Blindern, Oslo, Norway
}

Received August 31; in revised form December 5, 1972

\begin{abstract}
We consider quantum field theoretical models in $n$ dimensional space-time given by interaction densities which are bounded functions of an ultraviolet cut-off boson field. Using methods of euclidean Markov field theory and of classical statistical mechanics, we construct the infinite volume imaginary and real time Wightman functions as limits of the corresponding quantities for the space cut-off models. In the physical Hilbert space, the space-time translations are represented by strongly continuous unitary groups and the generator of time translations $H$ is positive and has a unique, simple lowest eigenvalue zero, with eigenvector $\Omega$, which is the unique state invariant under space-time translations. The imaginary time Wightman functions and the infinite volume vacuum energy density are given as analytic functions of the coupling constant. The Wightman functions have cluster properties also with respect to space translations.
\end{abstract}

\section{Introduction}

In recent years the mathematical construction of quantum field theoretical models has made an impressive progress ${ }^{1}$. For the polynomial interactions ${ }^{2}$ in two-dimensional space-time all the HaagKastler axioms for a quantum field theory of local observables have been verified, as well as most of the Wightman axioms ${ }^{3}$.

In particular in these polynomial models (and also for certain 2-dimensional boson models with exponential interactions [4]) the existence of a vacuum state has been proven ${ }^{4}$.

This was sufficient for Glimm and Jaffe to build a theory in which the Wightman functions exist and have some of the important physical properties embodied in Wightman's axioms.

The question of the uniqueness of the vacuum has not been tackled yet. The vacuum state is only obtained by a compactness argument as

\footnotetext{
${ }^{1}$ See e.g. [1] and the references given therein.

2 See e.g. $[1,2]$ and the references given therein.

${ }^{3}$ See e.g. [1-3]. See also footnote 5 below.

4 This has been proven also for the two-dimensional Yukawa interaction [5].
} 
limit of a subsequence of space cut-off vacua, so that the possibility of different subsequences giving rise to different vacua is not ruled out ${ }^{5}$.

In this paper we would like to remark that for certain non polynomial interactions in $n$ space-time dimensions with ultraviolet cut-off but no space cut-off uniqueness of the vacuum can be proven for small values of the coupling constant. Moreover the corresponding Wightman functions can be constructed and studied. The formal Hamiltonian of the boson models which we study has the form

$$
H_{0}+\lambda \int_{\mathbb{R}^{n-1}} e^{i s \varphi_{\varepsilon}(\boldsymbol{x})} d v(s) d \boldsymbol{x},
$$

where $\varphi_{\varepsilon}$ is an ultraviolet cut-off, free, time zero, field and $d v(s)$ is a measure with bounded support on the real line (and $d v(-s)=d v \bar{s})$, meaning complex conjugate) ${ }^{6}$.

We first prove that the space cut-off Schwinger functions (imaginary time Wightman functions) have unique limits when the space cut-off is removed, provided the coupling constant $\lambda$ is sufficiently small. These limit Schwinger functions are given explicitely in terms of LiouvilleNeumann series with known kernel as convergent power series in $\lambda$. Moreover they have cluster properties with respect to space and time translations. For real $\lambda$, with $|\lambda|$ sufficiently small, the Schwinger functions are analytic in the upper half planes of suitable time differences and their boundary values are the infinite volume Wightman functions, which are limits in the sense of distributions of the Wightman functions for the space cut-off interaction. The infinite volume Wightman functions, which satisfy the positive definiteness conditions, yield then the physical Hilbert space $\mathscr{H}$, with a cyclic vector $\Omega$ and a representation of the field operators by symmetric operators on an invariant domain and a strongly continuous unitary representation of the space-time translations.

The generator $H$ of the time translations is non negative and, due to cluster properties of the Wightman functions, has zero as a simple lowest eigenvalue, with eigenvector $\Omega . \Omega$ is the only state in $\mathscr{H}$ which is invariant under space-time translations. The Wightman functions are also proved to have the cluster property with respect to translations in space. A connection of the vacuum state with the limit, as the space cut-off is

${ }_{5}$ After completion of this paper we learned in a private communication from Glimm that for the polynomial interactions in two space-time dimensions without cut-offs he and collaborators (Dimock and Spencer) have solved the problem of the uniqueness of the vacuum for small coupling constants. As far as we know this has been done by methods different from the one we use in the present paper.

${ }^{6}$ These models are related to the bounded interaction models studied in [6]. They are, in a sense, an Hamiltonian version of certain "non polynomial interactions" studied in recent years from other points of view. See e.g. [7]. 
taken away, of the space cut-off vacuum state on an algebra of operators defined in terms of the time $C^{*}$-automorphism is also given.

The limit $\tilde{\varepsilon}$ of the ground state energy densities of the space cut-off Hamiltonians exists, is analytic in $\lambda$ for $|\lambda|$ small and concave in $\lambda$. It also exists for arbitrary negative $\lambda$ and positive $d v$ and $\tilde{\varepsilon}$ is then negative, decreasing for $|\lambda|$ increasing and concave in $\ln (-\lambda)$.

The idea of the proofs is suggested by the analogy between euclidean field theory and classical statistical mechanics, on one hand ${ }^{8}$ and, on the other hand, by the relation between Minkowski quantum field theory and euclidean Markov field theory as recently established by Nelson [9] ${ }^{9}$.

\section{The Space Cut-off Models}

Let $\mathscr{F}$ be the Fock space for free, scalar, uncharged bosons of strictly positive mass $m$, moving in $n$ dimensional space-time. Thus $\mathscr{F}$ is the direct sum $\mathscr{F}=\bigoplus_{r=0}^{\infty} \mathscr{F}^{(r)}$, where $\mathscr{F}^{(0)} \equiv \mathbb{C}=$ complex number and $\mathscr{F}^{(r)}$, for $r=1,2, \ldots$, is the $r$-fold symmetric tensor product $\mathscr{\mathscr { H }}^{(r)}=\mathscr{H} \underset{s}{\otimes} \cdots \underset{s}{\otimes} \mathscr{H}$, $\mathscr{H}$ being the Lebesgue $L^{2}$-space of (equivalence classes of) functions of a (momentum) variable $p$ running over the euclidean $n-1$ dimensional space $\mathbb{R}^{n-1}$.

Let $H_{0}$ be the free Hamiltonian in $\mathscr{F}$. It is a self-adjoint operator with domain $D\left(H_{0}\right) \equiv D_{0}$.

For $\boldsymbol{x}$ in $\mathbb{R}^{n-1}$ the free time zero fields are given by

$$
\varphi(\boldsymbol{x})=2^{-\frac{1}{2}}(2 \pi)^{-\frac{n-1}{2}} \int_{\mathbb{R}^{n-1}} \frac{e^{\imath \boldsymbol{p} \boldsymbol{x}}}{\mu(\boldsymbol{p})^{\frac{1}{2}}}\left[a^{*}(-\boldsymbol{p})+a(\boldsymbol{p})\right] d \boldsymbol{p},
$$

7 Note that, due to the presence of the ultraviolet cut-off, no Wick ordering of the interaction is required. In fact our interactions

$$
\int e^{\imath \varsigma \varphi_{\varepsilon}(\boldsymbol{x})} d v(s) d \boldsymbol{x}
$$

and the correspondent Wick-ordered ones

$$
\int: e^{i s \varphi_{\ell}(\boldsymbol{x})}: d v_{1}(s) d \boldsymbol{x}
$$

can be made to coincide by choosing $d v_{1}(s)=\exp \left(-\frac{1}{2} s^{2} K\right) d v(s)$, where $K$ is a constant (equal to the value for $x=0$ of the propagator $G_{\varepsilon}(x)$ defined below).

8 This analogy has been exploited from a different point of view particularly in the references [8] (and references quoted therein) and [7b,c].

9 See also [10], where a euclidean Markov field theoretical relation is exploited to prove the uniqueness of the vacuum energy density and the van Hove phenomenon for two-dimensional polynomial interactions. For further results on this infinite volume behaviour, see [11]. For references concerning work previous to Nelson's one, see [8]. 
where $\mu(\boldsymbol{p})=\sqrt{\boldsymbol{p}^{2}+m^{2}} . a(\boldsymbol{p})$ and $a^{*}(\boldsymbol{p})$ are the usual formal annihilationcreation operators for free scalar, uncharged bosons, normalized so that $\left[a(\boldsymbol{p}), a^{*}\left(\boldsymbol{p}^{\prime}\right)\right] \equiv a(\boldsymbol{p}) a^{*}\left(\boldsymbol{p}^{\prime}\right)-a^{*}\left(\boldsymbol{p}^{\prime}\right) a(\boldsymbol{p})=\delta\left(\boldsymbol{p}-\boldsymbol{p}^{\prime}\right)$.

Let $\chi(x)$ be a positive symmetric $C^{\infty}$ function in $\mathbb{R}^{n-1}$ with support in the unit ball such that $\int \chi(\boldsymbol{x}) d \boldsymbol{x}=1$. Set $\chi_{\varepsilon}=\varepsilon^{-n+1} \chi\left(\varepsilon^{-1} \boldsymbol{x}\right)$, with $\varepsilon>0$, and define the ultraviolet cut-off free time zero field by

$$
\varphi_{\varepsilon}(\boldsymbol{x})=\int \varphi(\boldsymbol{y}) \chi_{\varepsilon}(\boldsymbol{x}-\boldsymbol{y}) d \boldsymbol{y} .
$$

Then $\varphi_{\varepsilon}(\boldsymbol{x})$ are self-adjoint operators in $\mathscr{F}$ with definition domain containing $D_{0}$ and they are essentially self-adjoint on $D_{0}$. They are bounded from $\mathscr{F}^{(r)}$ into $\mathscr{F}^{(r-1)} \oplus \mathscr{F}^{(r+1)}$.

Let now $v(\alpha)$ be a real-valued function on $\mathbb{R}$, so chosen as to be the Fourier transform of a finite measure $d v$ of bounded support on the real line:

$$
v(\alpha)=\int e^{i s x} d v(s)
$$

with $\int d|v|<\infty$ and $v(-s)=\overline{v(s)}$.

The interaction density is given by $\lambda v\left(\varphi_{\varepsilon}(\boldsymbol{x})\right)$, which is a well defined bounded self-adjoint operator since $v(\alpha)$ is a bounded continuous function.

We note that

$$
v\left(\varphi_{\varepsilon}(\boldsymbol{x})\right)=\int e^{i \varsigma \varphi_{\varepsilon}(\boldsymbol{x})} d v(s),
$$

where the integral is taken in the strong sense. This is of the same form as the bounded interaction densities studied in [6].

The space cut-off interaction corresponding to this interaction density is given by

$$
\lambda V_{l} \equiv \lambda \int_{|\boldsymbol{x}| \leqq l} v\left(\varphi_{\varepsilon}(\boldsymbol{x})\right) d \boldsymbol{x},
$$

where the integral is again to be understood as a strong one. This defines $\lambda V_{l}$ as a bounded self-adjoint operator on $\mathscr{F}$ for all $l$.

Hence $H_{l} \equiv H_{0}+\lambda V_{l}$ is a self-adjoint operator, bounded from below, with the same domain $D_{0}$ as $H_{0}$.

Moreover we have from [6c] (Theorem 3) that, for arbitrary $\lambda$, the bottom of the spectrum of $H_{l}$ consists of the simple eigenvalue $E_{l}$ with (unique) eigenvector $\Omega_{l}{ }^{10}$.

From regular perturbation theory alone one has the additional result (which we are going to extend, in a certain sense, also for $l \rightarrow \infty$ ) that for

${ }^{10} E_{l}$ and $\Omega_{l}$ are obtained in [6c] as the unique (norm) limits of the lowest eigenvalues and respective eigenvectors of suitable approximating Hamiltonians ("piecewise constant momentum approximation"). 
$|\lambda|$ sufficiently small (depending on l) $E_{l}$ and $\Omega_{l}$ are analytic in $\lambda$. Moreover $E_{l}$ is a concave function of $\lambda$ i.e. satisfies $E_{l}\left(\alpha \lambda_{1}+(1-\alpha) \lambda_{2}\right) \geqq \alpha E_{l}\left(\lambda_{1}\right)$ $+(1-\alpha) E_{l}\left(\lambda_{2}\right)$ for all $0 \leqq \alpha \leqq 1, \lambda_{1}, \lambda_{2}$.

\section{The Associated Euclidean Markov Field}

For any real Hilbert space $\mathscr{H}$ let $\Phi_{\mathscr{H}}(h), h \in \mathscr{H}$ be the Gaussian generalized stochastic process indexed by $\mathscr{H}$, with mean zero and covariance $E\left(\Phi_{\mathscr{H}}(g) \Phi_{\mathscr{H}}(h)\right)=(g, h)_{\mathscr{H}}{ }^{11}$. So that $\Phi_{\mathscr{H}}(h)$ maps $h \in \mathscr{H}$ into a measurable function (Gaussian random variable) on a probability space $\left(\Omega_{\mathscr{H}}, d \mu_{\mathscr{H}}\right)$. Let $L_{2}\left(d \mu_{\mathscr{H}}\right)$ be the $L^{2}$-space over $\Omega_{\mathscr{P}}$ with respect to the measure $d \mu_{\mathscr{H}} . L_{2}\left(d \mu_{\mathscr{H}}\right)$ is isomorphic $[13,14]$ with the Fock space $\bigoplus_{n=0}^{\infty} \mathscr{H}^{(n)}$ over $\mathscr{H}$, where $\mathscr{H}^{(n)}$ is the $n$-fold symmetric tensorproduct of $\mathscr{H}$. Using this isomorphism we see that any strongly continuous unitary group on $\mathscr{H}$ induces, through a group of measure preserving transformations on $\Omega_{\mathscr{H}}$, a strongly continuous unitary group on $L_{2}\left(d \mu_{\mathscr{H}}\right)$.

Let $\Delta$ be the Laplacian as a self-adjoint operator in $L_{2}\left(\mathbb{R}^{n}\right)$. Let $\mathscr{H}_{n}^{\alpha}$ be the real Sobolev space, which is the completion of $C_{0}^{\infty}\left(\mathbb{R}^{n}\right)$ with respect to the inner product in $\mathscr{H}_{n}^{\alpha}$ given by

$$
(f, g)_{\alpha}=\left(f,\left(-\Delta+m^{2}\right)^{\alpha} g\right),
$$

where (, ) is the inner product in $L_{2}\left(\mathbb{R}^{n}\right)$, and $m$ is chosen to be the mass of the free field discussed in Section 2. For $\alpha<0, \mathscr{H}_{n}^{\alpha}$ will be a space of distributions.

The generalized Gaussian stochastic process $\Phi_{\mathscr{H}_{n}^{-1}}(h)$ is called the free euclidean Markov field. Using ideas introduced by Nelson [9] in the constructive study of models, we associate to the free time zero field over $\mathbb{R}^{n-1}, \varphi(g)=\int \varphi(\boldsymbol{x}) g(\boldsymbol{x}) d \boldsymbol{x}$ of Section 2, the euclidean Markov field $\Phi_{\mathscr{H}^{-1}}(h)$.

For any open set $U$ with smooth boundary in $\mathbb{R}^{n}$ let $\mathcal{O}(U)$ be the family of random variables generated by $\Phi(h)$, with $h \in \mathscr{H}_{n}^{-1}$ and support of $h$ in $U$. Let $E\{\Phi(h) \mid \mathcal{O}(U)\}$ be the conditional expectation of $\Phi(h)$ given $\mathcal{O}(U)$. Nelson proved that $\Phi(h)$ has the following "Markovian property":

$$
E\{\Phi(h) \mid \mathcal{O}(\mathcal{C} U)\}=E\{\Phi(h) \mid \mathcal{O}(\partial U)\}
$$

where $C U$ is the complement of $U$ and $\partial U$ is the boundary. The property (3.2) is taken as the characterizing property of a Markov field.

The Fock space of the free boson field as given in Section 2 is just the Fock space over $\mathscr{H}_{n-\frac{1}{1}}^{-\frac{1}{1}}$, moreover the free time zero field itself $\varphi(g)$

\footnotetext{
11 See e.g. [12].
} 
is a generalized Gaussian stochastic process with mean zero and covariance function

$$
E(\varphi(f) \varphi(g))=(f, g)_{-\frac{1}{2}} .
$$

Hence the free time zero field $\varphi(g)$ may be identified with the generalized Gaussian stochastic process $\Phi_{\mathscr{H}_{n}^{-\frac{1}{2}} 1}(g)$.

We define now a mapping $W_{t}: \mathscr{H}_{n-1}^{-\frac{1}{2}} \rightarrow \mathscr{H}_{n}^{-1}$ by $\left(W_{t} f\right)(x)$ $=\delta\left(x_{0}-t\right) f(\boldsymbol{x})$. One verifies easily that $W_{t}$ is an isometry of $\mathscr{H}_{n-1}^{-\frac{1}{2}}$ onto the closed subspace of $\mathscr{H}_{n}^{-1}$ generated by elements of $\mathscr{H}_{n}^{-1}$ with support on the hyperplane $x_{0}=t$.

The Fock space of the free boson field, $\mathscr{F}$, is the Fock space over $\mathscr{H}_{n-\frac{1}{1}}^{-\frac{1}{2}}$, hence identified with $L_{2}\left(d \mu_{\mathscr{H}_{n}^{-\frac{1}{2}}}\right)$. Since $W_{0}$ is an isometry, we have that the generalized Gaussian stochastic processes $\Phi_{\mathscr{H}_{n}^{-\frac{1}{2}}{ }_{1}}(g)$ and $\Phi_{\mathscr{H}_{n}^{-1}}\left(W_{0} g\right)$ have the same mean and covariance functions, hence may be identified. This then identifies $L_{2}\left(d \mu_{\mathscr{H}_{n}^{-\frac{1}{2}}}\right)$ with a closed subspace of $L_{2}\left(d \mu_{\mathscr{H}_{n}^{-1}}\right)$.

Let $F \in L_{2}\left(d \mu_{\mathscr{H}_{n}^{-\frac{1}{2}}}\right)$ be of the form $F=f\left(\Phi_{\mathscr{H}_{n}^{-\frac{1}{2}}-1}\left(g_{1}\right), \ldots, \Phi_{\mathscr{H}_{n}^{-\frac{1}{2}}}\left(g_{k}\right)\right)$, where $f$ is a bounded continuous function of $k$ real variables. Then we define $F_{t} \in L_{2}\left(d \mu_{\mathscr{H}_{n}^{-1}}\right)$ by $F_{t}=f\left(\Phi_{\mathscr{H}_{n}^{-1}}\left(W_{t} g_{1}\right), \ldots, \Phi_{\mathscr{H}_{n}^{-1}}\left(W_{t} g_{k}\right)\right)$. Using that $W_{t}$ is an isometry one gets that $F \rightarrow F_{t}$ extends to an isometry of $L_{2}\left(d \mu_{\mathscr{H}_{n}^{-\frac{1}{2}}}\right)$ into $L_{2}\left(d \mu_{\mathscr{H}_{n}^{-1}}\right)$. Moreover in $\mathscr{H}_{n}^{-1}$ the translation group acts unitarily and strongly continuously. Using the identification of $L_{2}\left(d \mu_{\mathscr{H}_{n}^{-1}}\right)$ with the Fock space over $\mathscr{H}_{n}^{-1}$ we get a unitary and strongly continuous representation $U(x)$ of the translation group in $\mathbb{R}^{n}$ on $L_{2}\left(d \mu_{\mathscr{H}_{n}^{-1}}\right)$. Since $F_{t}=U(t, \mathbf{0}) F_{0} U(-t, \mathbf{0})$, we see that $F_{t}$ depends continuously on $t$ in the $L_{2}$-norm for any $F$ in $L_{2}\left(d \mu_{\mathscr{H}_{n}^{-\frac{1}{2}}}\right)$.

One verifies that

$$
E\left(\Phi_{\mathscr{H}_{n}^{-1}}\left(h_{1}\right) \ldots \Phi_{\mathscr{H}_{n}^{-1}}\left(h_{r}\right)\right)=\left\{\begin{array}{l}
\sum_{\text {all partitions }}\left(h_{n_{1}}, h_{n_{2}}\right)_{-1} \ldots\left(h_{n_{r-1}}, h_{n_{r}}\right)_{-1} \\
n_{1}<n_{2}, \ldots, n_{r-1}<n_{r} \\
0 \text { for } r \text { odd }
\end{array}\right.
$$

from which it follows that the distributions of $r$-variables defined by $E\left(\Phi_{\mathscr{H}_{n}^{-1}}\left(h_{1}\right) \ldots \Phi_{\mathscr{H}_{n}^{-1}}\left(h_{r}\right)\right)$ are the imaginary time free field Wightman functions. Hence, for $t_{1} \leqq t_{2} \ldots \leqq t_{r}$,

$$
\begin{aligned}
& E\left(\Phi_{\mathscr{H}_{n}^{-1}}\left(W_{t_{1}} g_{1}\right) \ldots \Phi_{\mathscr{H}_{n}^{-1}}\left(W_{t_{r}} g_{r}\right)\right) \\
& \quad=\left(\Omega_{0}, \varphi\left(g_{1}\right) e^{-\left(t_{2}-t_{1}\right) H_{0}} \varphi\left(g_{2}\right) e^{-\left(t_{3}-t_{2}\right) H_{0}} \ldots \varphi\left(g_{r}\right) \Omega_{0}\right),
\end{aligned}
$$

where $\Omega_{0} \in \mathscr{F}$ is the vacuum for the free scalar boson field and $H_{0}$ is the free energy. Using now the identification of $\mathscr{F}$ with $L_{2}\left(d \mu_{\mathscr{H}_{n}^{-\frac{1}{2}}}\right)$ and taking 
sums and limits of expressions of the form (3.5) we get the following lemma.

Lemma 3.1. Let $F^{(1)}, \ldots, F^{(r)}$ be in $L_{\infty}\left(d \mu_{\mathscr{H}_{n}^{-\frac{1}{2}}}\right)$.

Then, for $t_{1} \leqq \cdots \leqq t_{r}$,

$$
E\left(F_{t_{1}}^{(1)} \ldots F_{t_{r}}^{(r)}\right)=\left(\Omega_{0}, F^{(1)} e^{-\left(t_{2}-t_{1}\right) H_{0}} F^{(2)} e^{-\left(t_{3}-t_{2}\right) H_{0}} \ldots F^{(r)} \Omega_{0}\right) .
$$

We will now consider self-adjoint operators of the form $H=H_{0}+V$, where $H_{0}$ is the free energy and $V$ is a bounded operator on $\mathscr{F}$ which commutes with all the free time zero fields $\varphi(g)$. Since the $L_{2}\left(d \mu_{\mathscr{H}_{n}^{-\frac{1}{2}}}\right)$ is a spectral representation of $\mathscr{F}$ with respect to the maximal abelian algebra generated by $\varphi(g)$, we see that, in $L_{2}\left(d \mu_{\mathscr{H}_{n}^{-\frac{1}{2}}}\right), V$ is a multiplication operator by a function, which we will also denote $V$.

Lemma 3.2. Let $V$ be as above, and let $F$ and $G$ be in $L_{2}\left(d \mu_{\mathscr{H}_{n}^{-\frac{1}{2}}}\right)$, then

$$
E\left(F_{0} e^{-\int_{0}^{t} V_{\tau} d \tau} G_{t}\right)=\left(\Omega_{0}, F e^{-t\left(H_{0}+V\right)} G \Omega_{0}\right)
$$

where the integral over $V_{\tau}$ is taken in the strong $L_{2}\left(d \mu_{\mathscr{H}_{n}^{-1}}\right)$ sense.

Proof. The Trotter product formula gives us

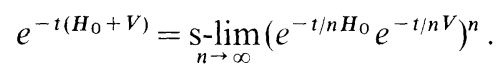

Now, by Lemma 3.1,

$\left(\Omega_{0}, F e^{-t / n H_{0}} e^{-t / n V} \ldots e^{-t / n H_{0}} e^{-t / n V} G \Omega_{0}\right)=E\left(F_{0} e^{-t / n \sum_{k=1}^{n} V_{k t / n}} G_{t}\right)$.

Since $V$ is in $L_{\infty}\left(d \mu_{\mathscr{H}_{n}^{-\frac{1}{2}}}\right)$ we know that $V_{t}$ is in $L_{\infty}\left(d \mu_{\mathscr{H}_{n}^{-1}}\right)$ and is continuous in $t$ in the strong $L_{2}$-sense. Hence $t / n \sum_{k=1}^{n} V_{k t / n}$ converges strongly in $L_{2}\left(d \mu_{\mathscr{H}_{n}^{-1}}\right)$ to $\int_{0}^{t} V_{\tau} d \tau$ for $n \rightarrow \infty$.

The strong $L_{2}$-convergence allows us to conclude that any subsequence has a subsequence $n_{j}$ such that the convergence is almost everywhere. The almost everywhere convergence together with the uniform boundedness gives that

$$
E\left(F_{0} e^{-t / n_{j} \sum_{k=1}^{n_{j}} V_{k t / n_{j}}} G_{t}\right) \underset{j \rightarrow \infty}{\longrightarrow} E\left(F_{0} e^{-\int_{0}^{t} V_{\tau} d \tau} G_{t}\right)
$$

This implies that the right hand side of (3.6) converges to $E\left(F_{0} e^{-\int_{0}^{t} V_{\tau} d \tau} G_{t}\right)$, which proves the lemma. 
The interaction of Section 2,

$$
\lambda V_{l}=\lambda \int_{|\boldsymbol{x}| \leqq l} v\left(\varphi_{\varepsilon}(\boldsymbol{x})\right) d \boldsymbol{x},
$$

is of the form considered in Lemma 3.2. Moreover the function $V_{\tau}$ in $L_{\infty}\left(d \mu_{\mathscr{H}_{n}^{-1}}\right)$ of Lemma 3.2 may be given explicitely in this case:

$$
V_{\tau}=\lambda \int_{|\boldsymbol{x}| \leqq l} v\left(\Phi_{\mathscr{H}_{n}^{-1}}\left(f_{\tau, \boldsymbol{x}}\right)\right) d \boldsymbol{x},
$$

where $f_{\tau, \boldsymbol{x}}(y)=\delta\left(\tau-y_{0}\right) \chi_{\varepsilon}(\boldsymbol{x}-\boldsymbol{y})$. This follows from the identification of $\varphi(g)$ with $\Phi_{\mathscr{H}_{n}^{-\frac{1}{2}} 1}(g)$ and the definition of the mapping $F \rightarrow F_{t}$ from $L_{2}\left(d \mu_{\mathscr{H}_{n}^{-\frac{1}{2}}}\right)$ into $L_{2}\left(d \mu_{\mathscr{H}_{n}^{-1}}\right)$. Since

$$
v\left(\Phi_{\mathscr{H}_{n}^{-1}}\left(f_{\tau, \boldsymbol{x}}\right)\right)=U(-\tau,-\boldsymbol{x}) v\left(f_{0, \boldsymbol{0}}\right) U(\tau, \boldsymbol{x}),
$$

we see that the integrand in (3.8) is continuous in $\boldsymbol{x}$ as well as in $\tau$ in the strong $L_{2}$-sense. Hence in this case Lemma 3.2 takes the form

Lemma 3.3. Let $v(\alpha)$ be as in Section 2. Then

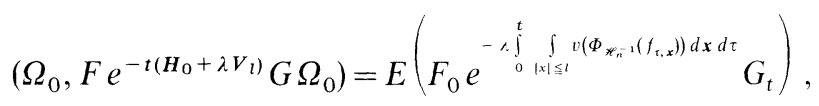

where $F$ and $G$ are in $L_{2}\left(d \mu_{\mathscr{H}_{n}^{-\frac{1}{2}}}\right)$, and $f_{\tau, \boldsymbol{x}}(y)=\delta\left(\tau-y_{0}\right) \chi_{\varepsilon}(\boldsymbol{x}-\boldsymbol{y})$.

From (3.4) it follows that $\Phi_{\mathscr{H}_{n}^{-1}}(h)$ for $h \in \mathscr{H}_{n}^{-1}$ is in all $L_{p}$ for $1 \leqq p<\infty$. For $V$ in $L_{\infty}\left(d \mu_{\mathscr{H}_{n}^{-\frac{3}{2}}}\right)$ we may therefore consider $E\left(\Phi\left(h_{1}\right) \ldots \Phi\left(h_{n}\right) e^{-\int_{a}^{b} V_{\tau} d \tau}\right)$, where we have written $\Phi(h)$ for $\Phi_{\mathscr{H}_{n}^{-1}}(h)$. Take $h_{1}, \ldots, h_{n}$ in $C_{0}^{\infty}\left(\mathbb{R}^{n}\right)$ and set $g_{i}^{t}(\boldsymbol{x})=h_{i}(t, \boldsymbol{x})$.

Then $h_{i}\left(x_{0}, \boldsymbol{x}\right)=\int \delta\left(x_{0}-t\right) g_{i}^{t}(\boldsymbol{x}) d t$ and the integrand $\delta\left(x_{0}-t\right) g_{i}^{t}(\boldsymbol{x})$ is strongly continuous in $\mathscr{H}_{n}^{-1}$. Therefore if the support of $h_{i}$ is bounded by the hyperplanes $x_{0}=a$ and $x_{0}=b$, then

$$
\begin{aligned}
& E\left(\Phi\left(h_{1}\right) \ldots \Phi\left(h_{n}\right) e^{-\int_{a}^{b} V_{\tau} d \tau}\right) \\
=n ! & \int_{a \leqq t_{1} \leqq \cdots \leqq t_{n} \leqq b} E\left(\Phi\left(W_{t_{1}} g_{1}^{t_{1}}\right) \ldots \Phi\left(W_{t_{n}} g_{n}^{t_{n}}\right) e^{-\int_{a}^{b} V_{\tau} d \tau}\right) d t_{1} \ldots d t_{n},
\end{aligned}
$$

which by formula (3.5) and Lemma 3.2 is equal to

$$
\begin{gathered}
n ! \int_{a \leqq t_{1} \leqq \cdots \leqq} \cdots \int_{t_{n} \leqq b}\left(\Omega_{0}, e^{-\left(t_{1}-a\right) H} \varphi\left(g_{1}^{t_{1}}\right) e^{-\left(t_{2}-t_{1}\right) H} \varphi\left(g_{2}^{t_{2}}\right) \ldots\right. \\
\left.\ldots \varphi\left(g_{n}^{t_{n}}\right) e^{-\left(b-t_{n}\right) H} \Omega_{0}\right) d t_{1} \ldots d t_{n},
\end{gathered}
$$

with $H=H_{0}+V$. 
Let $E$ be the infimum of the spectrum of $H$, and set $\bar{H}=H-E$. Since $V$ is bounded we have

$$
\left.\| \varphi\left(g_{i}^{t_{i}}\right) e^{-\left(t_{2}+1-t_{1}\right.}\right)^{\bar{H}}\|\leqq C\|(\bar{H}+1)^{\frac{1}{2}} e^{-\left(t_{1}+1-t_{2}\right) \bar{H}} \|,
$$

with $C$ independent of $t_{i}$ and $i$. On the other hand for any positive selfadjoint operator $A$ we have

$$
\left\|(A+1)^{\frac{1}{2}} e^{-t A}\right\| \leqq \sup _{x>0}(x+1)^{\frac{1}{2}} e^{-t x}=(2 t)^{-\frac{1}{2}} e^{t-\frac{1}{2}} .
$$

Using (3.11), (3.12) and the fact that $\varphi\left(g_{i}^{t}\right)$ is zero for $t$ outside a bounded interval, we get that

$$
\left(\Omega_{0}, e^{-\left(t_{1}-a\right) \bar{H}} \varphi\left(g_{1}^{t_{1}}\right) e^{-\left(t_{2}-t_{1}\right) \bar{H}} \ldots \varphi\left(g_{n}^{t_{n}}\right) e^{-\left(b-t_{n}\right) \bar{H}} \Omega_{0}\right)
$$

is bounded in absolute value uniformly in $a$ and $b$ by an integrable function over $t_{1} \leqq \cdots \leqq t_{n}$.

Let us assume that $H$ has a simple eigenvalue at $E$ and let $\Omega$ be the corresponding eigenvector. Then $e^{-\left(t_{1}-a\right) \bar{H}} \Omega_{0}$ as well as $e^{-\left(b-t_{n}\right) \bar{H}} \Omega_{0}$ converge to $\left(\Omega, \Omega_{0}\right) \Omega$ as $a \rightarrow-\infty$ and $b \rightarrow+\infty$. By (3.11) $\varphi\left(g_{i}^{t_{2}}\right) e^{-\left(t_{1}+1-t_{t}\right) \bar{H}}$ is a bounded operator for $t_{1}<t_{2}<\cdots<t_{n}$. Hence (3.13) converges to

$$
\left|\left(\Omega, \Omega_{0}\right)\right|^{2}\left(\Omega, \varphi\left(g_{1}^{t_{1}}\right) e^{-\left(t_{2}-t_{1}\right) \bar{H}} \ldots e^{-\left(t_{n}-t_{n-1}\right) \bar{H}} \varphi\left(g_{n}^{t_{n}}\right) \Omega\right)
$$

as $a \rightarrow-\infty$ and $b \rightarrow+\infty$ for $t_{1}<t_{2}<\cdots<t_{n}$.

From Lebesgue's dominated convergence theorem we then get that $\left(\Omega_{0}, e^{-(b-a) H} \Omega_{0}\right)^{-1}$

$$
\cdot \int_{a \leqq t_{1} \leqq} \cdots \int_{t_{n} \leqq b}\left(\Omega_{0}, e^{-\left(t_{1}-a\right) H} \varphi\left(g_{1}^{t_{1}}\right) \ldots \varphi\left(g_{n}^{t_{n}}\right) e^{-\left(b-t_{n}\right) H} \Omega_{0}\right) d t_{1} \ldots d t_{n}
$$

converges to

$$
\int_{t_{1} \leqq \cdots \leqq t_{n}} \ldots \int_{t_{1}}\left(\Omega, \varphi\left(g_{1}^{t_{1}}\right) e^{-\left(t_{2}-t_{1}\right) \bar{H}} \ldots e^{-\left(t_{n}-t_{n}-1\right) \bar{H}} \varphi\left(g_{n}^{t_{n}}\right) \Omega\right) d t_{1} \ldots d t_{n}
$$

as $a \rightarrow-\infty$ and $b \rightarrow+\infty$. This proves the following Lemma.

Lemma 3.4. Let $h_{1}, \ldots, h_{n} \in C_{0}^{\infty}\left(\mathbb{R}^{n}\right)$, then

$$
\begin{aligned}
& \lim _{t \rightarrow+\infty}\left(E\left(e^{-\int_{-t}^{t} V_{t} d \tau}\right)\right)-1 \cdot E\left(\Phi\left(h_{1}\right) \ldots \Phi\left(h_{n}\right) e^{-\int_{-t}^{t} V_{\tau} d \tau}\right) \\
& =n ! \int_{t_{1} \leqq \cdots \leqq t_{n}} \ldots \int_{n}\left(\Omega, \varphi\left(g_{1}^{t_{1}}\right) e^{-\left(t_{2}-t_{1}\right) \bar{H}} \ldots e^{-\left(t_{n}-t_{n-1}\right) \bar{H}} \varphi\left(g_{n}^{t_{n}}\right) \Omega\right) d t_{1} \ldots d t_{n} .
\end{aligned}
$$

Remark 1. For $V=\lambda V_{l}$, the interaction of Section 2, this lemma holds since we know that $H_{l}=H_{0}+\lambda V_{l}$ has a simple lowest eigenvalue.

Remark 2. Lemma (3.4) shows that the limit is the time imaginary Wightman function for the space cut-off interaction integrated with $h_{1}\left(x_{1}\right) \ldots h_{n}\left(x_{n}\right)$. 


\section{Connection with Some Quantities of Classical Statistical Mechanics}

Let us denote the random variable $\Phi_{\mathscr{H}_{n^{-1}}}\left(f_{x_{0}, x}\right)$ by $\Phi_{\varepsilon}\left(x_{0}, x\right) \equiv \Phi_{\varepsilon}(x)$, where $f_{x_{0}, \boldsymbol{x}}(y)=\delta\left(x_{0}-y_{0}\right) \chi_{\varepsilon}(\boldsymbol{x}-\boldsymbol{y})$, and define for any bounded measurable $\Lambda \subset \mathbb{R}^{n}$ and for any $h_{1}, \ldots, h_{k}$ in $C_{0}\left(\mathbb{R}^{n}\right)$

and

$$
\begin{gathered}
Z_{\Lambda} \equiv E\left(e^{-\lambda \int_{\Lambda} v\left(\Phi_{\varepsilon}(x)\right) d x}\right), \\
F_{\Lambda}^{k}\left(h_{1}, \ldots, h_{k}\right) \equiv E\left(\Phi\left(h_{1}\right) \ldots \Phi\left(h_{k}\right) e^{-\lambda \int_{\Lambda} v\left(\Phi_{\varepsilon}(x)\right) d x}\right),
\end{gathered}
$$

$$
G_{A}^{k}\left(h_{1}, \ldots, h_{k}\right)=Z_{A}^{-1} F_{A}^{k}\left(h_{1}, \ldots, h_{k}\right) .
$$

From Lemma 3.4 we see that if we take $\Lambda=\Lambda_{t, l} \equiv\left\{x ;\left|x_{0}\right| \leqq t / 2,|x| \leqq l\right\}$, then the $G_{\Lambda_{t, l}}^{k}\left(h_{1}, \ldots, h_{k}\right)$ converge for $t \rightarrow \infty$ to the imaginary time Wightman functions for the space cut-off interaction. In order to remove the space cut-off we will therefore naturally be interested in taking the limit as $l \rightarrow \infty$ as well as $t \rightarrow \infty$ in $G_{\Lambda_{t, l}}^{k}$. We intend, by using methods from classical statistical mechanics, to prove that the limit of $G_{A}^{k}$ exists for $\Lambda$ expanding to $\mathbb{R}^{n}$. This will then give us the time imaginary Wightman functions for the model without cut-off.

So let $\Lambda$ be bounded. Since $v\left(\Phi_{\varepsilon}(x)\right)$ is a bounded random variable and strongly $L_{2}$-continuous in $x, Z_{A}$ and $F_{A}$ are entire functions of $\lambda$. Let us set

$$
F_{\Lambda}(h)=E\left(e^{i \Phi(h)} e^{-\lambda \int_{\Lambda} v\left(\Phi_{\varepsilon}(x)\right) d x}\right) \quad \text { and } \quad G_{\Lambda}(h)=Z_{\Lambda}^{-1} F_{\Lambda}(h) .
$$

Since $v\left(\Phi_{\varepsilon}(x)\right)$ is a bounded random variable we see from the definition of $F_{\Lambda}(h)$ that $F_{\Lambda}\left(\sum_{i=1}^{k} t_{i} h_{i}\right)$ is $k$ times differentiable with respect to $t_{1}, \ldots, t_{k}$ and that $\frac{\partial}{\partial t_{1}} \ldots \frac{\partial}{\partial t_{k}} F_{A}\left(\sum_{i=1}^{k} t_{i} h_{i}\right)=(i)^{k} F_{\Lambda}^{k}\left(h_{1}, \ldots, h_{k}\right)$ for $t_{1}=t_{2} \cdots=t_{k}=0$. Hence $F_{A}(h)$ determines $F_{A}^{k}\left(h_{1}, \ldots, h_{k}\right)$.

Since $v\left(\Phi_{\varepsilon}(x)\right)$ is a bounded random variable, $F_{A}(h)$ is also an entire function of $\lambda$. By expanding in powers of $\lambda$ we get

$$
F_{\Lambda}(h)=E\left(e^{i \Phi(h)}\right)+\sum_{n=1}^{\infty} \frac{(-\lambda)^{n}}{n !} \int \cdots \int E\left(e^{i \Phi(h)} v\left(\Phi_{\varepsilon}\left(x_{1}\right)\right) \ldots v\left(\Phi_{\varepsilon}\left(x_{n}\right)\right)\right) \prod_{j=1}^{n} d x_{j} .
$$

Using now that $v(\alpha)=\int e^{i s \alpha} d v(s)$ we get

$$
\begin{aligned}
& \int \underset{\Lambda^{n}}{\ldots \int} E\left(e^{i \Phi(h)} v\left(\Phi_{\varepsilon}\left(x_{1}\right)\right) \ldots v\left(\Phi_{\varepsilon}\left(x_{n}\right)\right)\right) \prod_{j=1}^{n} d x_{j} \\
& =\int \underset{A^{n}}{\ldots} E\left(e^{i\left(\boldsymbol{\Phi}(h)+\sum_{j=1}^{n} s_{j} \Phi_{\varepsilon}\left(x_{j}\right)\right)}\right) \prod_{j=1}^{n} d v\left(s_{j}\right) d x_{j} \\
& =\int \underset{A^{n}}{\cdots} E\left(e^{\left(i \Phi\left(h+\sum_{j=1}^{n} s_{j} f_{x_{j}}\right)\right.}\right) \prod_{j=1}^{n} d v\left(s_{j}\right) d x_{j},
\end{aligned}
$$


where $f_{x}(y)=\delta\left(x_{0}-y_{0}\right) \chi_{\varepsilon}(\boldsymbol{x}-\boldsymbol{y})$ by the definition of $\Phi_{\varepsilon}(x)$. On the other hand, for any $g \in \mathscr{H}_{n}^{-1}$

$$
E\left(e^{i \Phi(g)}\right)=e^{-\frac{1}{2}(g, g)-1}
$$

and setting

we get

$$
g=h+\sum_{j=1}^{n} s_{j} f_{x_{j}}
$$

$$
E\left(e^{\left(i \Phi\left(h+\sum_{j=1}^{n} s_{j} f_{x_{j}}\right)\right.}\right)=E\left(e^{i \Phi(h)}\right) e^{-\frac{1}{2} \sum_{l, j=1}^{n} s_{i} s_{j} G_{t}\left(x_{i}-x_{j}\right)-\sum_{j=1}^{n} s_{j} h^{\varepsilon}\left(x_{j}\right)},
$$

where $G_{\varepsilon}(x-y)=\left(f_{x}, f_{y}\right)_{-1}$ and $h^{\varepsilon}(x)=\left(h, f_{x}\right)_{-1}$.

Hence the integral over $\Lambda^{n}$ above is

$$
E\left(e^{i \Phi(h)}\right) \int \underset{A^{n}}{\int \ldots} e^{-\frac{1}{2} \sum_{l, j=1}^{n} s_{l} s_{j} G_{\varepsilon}\left(x_{l}-x_{j}\right)} \prod_{j=1}^{n}\left[\left(e^{-s_{j} h^{\varepsilon}\left(x_{J}\right)}-1\right)+1\right] \prod_{j=1}^{n} d v\left(s_{j}\right) d x_{j} .
$$

Computing now the product and using that $\sum_{i, l}^{n} s_{l} s_{j} G_{\varepsilon}\left(x_{i}-x_{j}\right)$ is symmetric under permutations of $x_{1} s_{1}, \ldots, x_{n} s_{n}$, we get this equal to

$$
E\left(e^{i \Phi(h)}\right) \sum_{r=0}^{n}\left(\begin{array}{l}
n \\
r
\end{array}\right) \cdot \int \underset{A^{n}}{\cdots} \int e^{-\frac{1}{2} \sum s_{i, j} s_{j} G_{\varepsilon}\left(x_{i}-x_{j}\right)} \prod_{j=1}^{r}\left(e^{-s_{j} h^{\varepsilon}\left(x_{j}\right)}-1\right) \prod_{j=1}^{n} d v\left(s_{j}\right) d x_{j} .
$$

From this it follows that

$$
\begin{gathered}
F_{\Lambda}(h)=E\left(e^{i \Phi(h)}\right)\left[Z_{\Lambda}+\sum_{r=1}^{\infty} \frac{1}{r !} \sum_{n=0}^{\infty} \frac{(-\lambda)^{n+r}}{n !} \int_{\Lambda^{n+r}} \cdots \int e^{-\frac{1}{2} \sum_{i, j=1}^{n+1} s_{l} s_{j} G_{\varepsilon}\left(x_{1}-x_{j}\right)}\right] \\
\cdot \prod_{j=1}^{r}\left(e^{-s_{j} h^{\varepsilon}\left(x_{j}\right)}-1\right) \prod_{j=1}^{n+r} d v\left(s_{j}\right) d x_{j}
\end{gathered}
$$

where we already have used that the expansion for $Z_{A}$ is given by

$$
Z_{\Lambda}=\sum_{n=0}^{\infty} \frac{(-\lambda)^{n}}{n !} \int \cdots_{\Lambda^{n}} \int e^{-\frac{1}{2} \sum_{i, j=1}^{n} s_{t} s_{j} G_{\varepsilon}\left(x_{t}-x_{j}\right)} \prod_{j=1}^{n} d v\left(s_{j}\right) d x_{j} .
$$

We remark that $G_{\varepsilon}(x)$ is a bounded real positive definite function, which tends to zero as $\frac{1}{|x|} e^{-m|x|}$ for $|x| \rightarrow \infty$. Since $G_{\varepsilon}(x)$ is positive definite, we have that $\left|G_{\varepsilon}(x)\right| \leqq G_{\varepsilon}(0)$. We notice that, for negative $\lambda, Z_{A}$ is in fact the grand canonical partition function for a gas in $n$-dimensional space with variably charged particles and activity $z=-\lambda$. The interaction energy between a particle at $x_{i}$ with charge $s_{i}$ and a particle at $x_{j}$ with 
charge $s_{j}$ is $s_{i} s_{j} G_{\varepsilon}\left(x_{i}-x_{j}\right)$, and the self energy of a particle with charge $s$ is given by $\frac{1}{2} s^{2} G_{\varepsilon}(0)$. So the charge $s$ is an internal degree of freedom for these particles, and $s$ may be discrete or continuous, depending on $d v$. We are going to exploit this connection with the grand canonical ensemble of a gas of variably charged particles, by introducing the corresponding correlation functions and we shall see that $G_{A}(h)$ can be expressed explicitely by these correlation functions ${ }^{12}$. The correlation functions $\varrho_{\Lambda}^{k}\left(x_{1} s_{1}, \ldots, x_{k} s_{k}\right)$ are defined for $x_{i} \in \mathbb{R}^{n}$ and $s_{t}$ in the support of $d v$ by

$$
\begin{aligned}
& \varrho_{A}^{k}\left(x_{1} s_{1}, \ldots, x_{k} s_{k}\right) \\
& \quad=Z_{A}^{-1} \sum_{n=0}^{\infty} \frac{(-\lambda)^{n+k}}{n !} \int{ }_{A^{n}} \ldots \int e^{-\frac{1}{2} \sum_{i, j=1}^{n+k} s_{l} s_{j} G_{s}\left(x_{i}-x_{j}\right)} \prod_{j=k+1}^{n+k} d v\left(s_{j}\right) d x_{j},
\end{aligned}
$$

for all $x_{\imath} \in \Lambda$ and zero elsewhere, for those values of $\lambda$ for which $Z_{A} \neq 0$. Since $\sum_{i, j} s_{i} s_{j} G_{\varepsilon}\left(x_{i}-x_{j}\right) \geqq 0$ we see that the series converge for all complex $\lambda$. From (4.1) it follows that $G_{A}(h)$ is given in terms of $\varrho_{A}^{k}$ by

$$
\begin{aligned}
& G_{\Lambda}(h)=e^{-\frac{1}{2}(h, h)-1} \\
& \cdot\left[1+\sum_{r=1}^{\infty} \frac{1}{r !} \int \ldots \int \prod_{A^{r}}^{r}\left(e^{-s_{j} h^{\varepsilon}\left(x_{j}\right)}-1\right) \varrho_{A}^{r}\left(x_{1} s_{1}, \ldots, x_{r} s_{r}\right) \prod_{j=1}^{r} d v\left(s_{j}\right) d x_{j}\right] .
\end{aligned}
$$

As in classical statistical mechanics ${ }^{13}$ we shall now introduce the Banach spaces $B_{\xi}$ of sequences $\psi=\left\{\psi_{k}\left((x s)_{k}\right)\right\}_{k \geqq 1}=\left\{\psi_{k}\left(x_{1} s_{1}, \ldots, x_{k} s_{k}\right)\right\}_{k \geqq 1}$ of bounded $d x d v$-measurable functions. The norm in $B_{\xi}$ is given by

$$
\|\boldsymbol{\psi}\|_{\xi}=\sup _{n} \xi^{-n} \underset{\substack{x_{1} \ldots x_{n} \\ s_{1} \ldots s_{n}}}{\operatorname{ess} \sup _{n} \mid \psi_{n}}\left(x_{1} s_{1}, \ldots, x_{n} s_{n}\right) \mid,
$$

where $\xi$ is a positive number.

In $B_{\xi}$ we define the projection operator $P_{A}$ of norm one given by

$$
\left(P_{A} \psi\right)(x s)_{n}=\chi_{A}(x)_{n} \psi(x s)_{n},
$$

12 The correlation functions of similar "euclidean gases of charged particles" associated with field theoretical models have been introduced, in another context, in Ref. [7b] and [7c].

13 These spaces have been introduced in classical statistical mechanics by Ruelle in order to study the infinite volume limit of the correlation functions in the grand canonical ensemble. Here and in the rest of this section we shall follow closely the lines of classical statistical mechanics as given in Ruelle's book, Ref. [15], Chapter 4. This reference contains also bibliographical notes on previous work on the infinite volume limit of correlation functions. 
where $\chi_{A}(x)_{n}=\chi_{A}\left(x_{1}\right) \ldots \chi_{A}\left(x_{n}\right)$, with $\chi_{A}(x)$ equal to the characteristic function for the set $A$. Also in analogy with statistical mechanics we introduce an operator $K$ on $B_{\xi}$ given by

$$
\begin{aligned}
(K \psi)(x s)_{m}= & e^{-\sum_{i=2}^{m} s_{1} s_{1} G_{\varepsilon}\left(x_{1}-x_{1}\right)} e^{-\frac{1}{2} s_{1}^{2} G_{\varepsilon}(0)}\left\{\psi_{m-1}\left(x_{2} s_{2}, \ldots, x_{m} s_{m}\right)\right. \\
& +\sum_{n=1}^{\infty} \frac{1}{n !} \int \cdots \int \prod_{j=1}^{n}\left[\left(e^{-s_{1} t_{j} G_{\varepsilon}\left(y_{j}-x_{1}\right)}-1\right)\right] \psi_{m+n-1} \\
& \left.\cdot\left(x_{2} s_{2}, \ldots, x_{m} s_{m}, y_{1} t_{1} \ldots y_{n} t_{n}\right) \prod_{j=1}^{n} d \nu\left(t_{j}\right) d y_{j}\right\} .
\end{aligned}
$$

For $m=1$ the first term in the curly bracket is set equal to zero.

Let $\boldsymbol{\alpha}$ be the sequence $\alpha_{1}\left(x_{1} s_{1}\right)=e^{-\frac{1}{2} s_{1}^{2} G_{\varepsilon}(0)}$ and $\alpha_{n}\left(x_{1} s_{1}, \ldots, x_{n} s_{n}\right)=0$ for $n>1$. We then verify that the sequence $\varrho_{A}$ given by the correlation functions $\varrho_{\Lambda}^{n}\left(x_{1} \ldots x_{n}\right)$ satisfies the equation

$$
\varrho_{\Lambda}=-\lambda P_{\Lambda} \alpha-\lambda P_{A} K \varrho_{\Lambda} .
$$

Since the correlation functions $\varrho_{A}^{n}\left(x_{1} s_{1}, \ldots, x_{n} s_{n}\right)$ are symmetric, we find from (4.7) that $\varrho_{\Lambda}$ will also satisfy the equation

$$
\varrho_{\Lambda}=-\lambda P_{\Lambda} \boldsymbol{\alpha}-\lambda P_{\Lambda} \Pi K \underline{\varrho}_{\Lambda},
$$

where $\Pi$ is an operator of the form

$$
(\Pi \psi)_{n}\left(x_{1} s_{1}, \ldots, x_{n} s_{n}\right)=\psi_{n}\left(x_{\sigma(1)} s_{\sigma(1)}, \ldots, x_{\sigma(n)} s_{\sigma(1)}\right),
$$

$\sigma$ being, for each $n$, a permutation of $1, \ldots, n$ which may depend measurably on $x_{1}, \ldots, x_{n}$ and $s_{1}, \ldots, s_{n}$.

We note that such a $\Pi$ will have norm equal to one.

Since $G_{\varepsilon}(x)$ is positive definite ${ }^{14}$ we have that

$$
\sum_{i \neq j}^{m} s_{i} s_{j} G_{\varepsilon}\left(x_{i}-x_{j}\right) \geqq-2 G_{\varepsilon}(0) \sum_{i=1}^{m} s_{i}^{2} .
$$

Let $B=G_{\varepsilon}(0) \sup \left\{s^{2} ; s \in \operatorname{supp}\right.$ of $\left.d v\right\}$; then

$$
\sum_{i \neq j}^{m} s_{i} s_{j} G_{\varepsilon}\left(x_{i}-x_{j}\right) \geqq-2 m B .
$$

It follows from (4.10) that for any $x_{1}, \ldots, x_{m}$ and $s_{1}, \ldots, s_{m}$ there exists an index $i$ such that

$$
\sum_{\substack{j=1 \\ j \neq i}}^{m} s_{i} s_{j} G_{\varepsilon}\left(x_{i}-x_{j}\right) \geqq-2 B .
$$

14 Using the analogy with classical statistical mechanics this can be interpreted as the fact that the total interaction of our gas in $\mathbb{R}^{n}$ satisfies the "stability condition" of [15]. 
For any $m$ and any $x_{1}, \ldots, x_{m}$ and $s_{1}, \ldots, s_{m}$ we now choose a permutation $\sigma$ of $1, \ldots, m$ such that $\sigma(1)=i$, where $i$ is the index $i$ of (4.11). $\sigma$ is then a permutation depending on the $x$ 's and the $s$ 's, and let $\Pi$ be the corresponding operator on $B_{\xi}$ defined by (4.9).

We now estimate the operator norm on $B_{\xi}$ of the operator $\Pi K$ of (4.8). From (4.6) and (4.11) we have

$$
\begin{aligned}
& \left|\Pi K \psi(x s)_{m}\right| \leqq e^{2 B}\left[\sup _{x, s}\left|\psi_{m-1}\left(x_{1} s_{1}, \ldots, x_{m-1} s_{m-1}\right)\right|\right] \\
& \quad+\sum_{n=1}^{\infty} \frac{1}{n !} C^{n} \sup _{x, s}\left|\psi_{m+n-1}\left(x_{1} s_{1}, \ldots, x_{m+n-1}, s_{m+n-1}\right)\right|
\end{aligned}
$$

with

$$
C=\sup _{s}\left\{\int\left|e^{-s t G_{\varepsilon}(x)}-1\right| e^{-\frac{1}{2} s^{2} G_{\varepsilon}(0)} d|v|(t) d x ; s \in \operatorname{supp} d v\right\} .
$$

It follows from the exponential decrease of $G_{\varepsilon}(x)$ that $C$ is finite ${ }^{15}$.

Using now that $\sup _{x, s}\left|\psi_{k}\left(x_{1} s_{1}, \ldots, x_{k} s_{k}\right)\right| \leqq \xi^{k}\|\boldsymbol{\psi}\|_{\xi}$, we get

Hence

$$
\left|(\Pi K \boldsymbol{\psi})_{m}(x s)_{m}\right| \leqq e^{2 B} \xi^{m-1} e^{\xi C} .
$$

$$
\|\Pi K \boldsymbol{\psi}\|_{\xi} \leqq \xi^{-1} e^{2 B+\xi C}\|\boldsymbol{\psi}\|_{\xi},
$$

so that $\|\Pi K\| \leqq C e^{2 B+1}$, if we choose $\xi=C^{-1}$, which is seen to be the best choice of $\xi$. This proves that (4.8) has a unique solution for $|\lambda|<C^{-1} e^{-2 B-1}$, which then is $\varrho_{A}$. From this we also get that the correlation functions $\varrho_{\Lambda}^{k}\left(x_{1} s_{1}, \ldots, x_{k} s_{k}\right)$ are analytic in $\lambda$ uniformly in $\Lambda$ for $|\lambda|<C^{-1} e^{-2 B-1}$. Moreover we may define $\varrho^{k}\left(x_{1} s_{1}, \ldots, x_{k} s_{k}\right)$ by

$$
\varrho=-\lambda \alpha-\lambda \Pi K \boldsymbol{\varrho}
$$

for $|\lambda|<C^{-1} e^{-2 B-1}$.

Lemma 4.1. For $|\lambda|<C^{-1} e^{-2 B-1}$ the infinite volume correlation functions $\varrho^{k}\left(x_{1} s_{1}, \ldots, x_{k} s_{k}\right)$ defined as the unique solution of (4.13) exist and are analytic in $\lambda^{16}$. Moreover they satisfy

$$
\left|\varrho^{k}\left(x_{1} s_{1}, \ldots, x_{k} s_{k}\right)\right| \leqq C^{-k} \frac{|\lambda|}{1-|\lambda| C e^{2 B+1}},
$$

are continuous in $x_{1}, \ldots, x_{k}$ and $s_{1} \ldots s_{k}$, and translation invariant in the $x$ 's. The finite volume correlation functions $\varrho_{A}^{k}\left(x_{1} s_{1}, \ldots, x_{k} s_{k}\right)$ converge to $\varrho^{k}\left(x_{1} s_{1}, \ldots, x_{k} s_{k}\right)$ as $\Lambda \rightarrow \mathbb{R}^{n}$ such that $d(x, \subset \Lambda) \rightarrow \infty$ for any $x \in \mathbb{R}^{n}$ and

${ }^{15}$ This can be interpreted as the fact that the interaction of our gas in $\mathbb{R}^{n}$ satisfies the "regularity condition" of [15].

16 Their expansions in powers of $\lambda$ are given as Liouville-Neumann series with known kernel: see Remark at the end of the Section 4. 
$d(x, \subset \Lambda)$ is the distance from $x$ to the complement of $\Lambda$. The convergence is such that

$$
\left|\varrho_{A}^{k}\left(x_{1} s_{1}, \ldots, x_{k} s_{k}\right)-\varrho^{k}\left(x_{1} s_{1}, \ldots, x_{k} s_{k}\right)\right| \leqq C^{-k} \eta(d),
$$

where $\eta$ is a function that goes to zero at infinity and is independent of $\Lambda, k$ and $x_{1} \ldots x_{k}, s_{1} \ldots s_{k}$, and $d=\min \left\{d\left(x_{i}, \complement \Lambda\right)\right\}$.

Lemma 4.2. For $|\lambda|<C^{-1} e^{-2 B-1}$ we have the cluster property for the correlation functions:

$$
\begin{aligned}
& \varrho^{k+l}\left(x_{1} s_{1}, \ldots, x_{k} s_{k}, y_{1}+a, t_{1}, \ldots, y_{l}+a, t_{l}\right) \\
\rightarrow & \varrho^{k}\left(x_{1} s_{1}, \ldots, x_{k} s_{k}\right) \varrho^{l}\left(y_{1} t_{1}, \ldots, y_{l} t_{l}\right),
\end{aligned}
$$

pointwise as a tends to infinity in $\mathbb{R}^{n}$.

These two Lemmas are proved as in classical statistical mechanics [15] by using that $s_{i} s_{j} G_{\varepsilon}\left(x_{i}-x_{j}\right)$ corresponds to a stable and regular interaction in the language of classical statistical mechanics. The proofs require only a slight modification of the proofs given in Ref. [15], Chapter 4 , and will therefore not be given here.

Lemma 4.3. For $|\lambda|<C^{-1} e^{-2 B-1}$ the limit

$$
\tilde{\varepsilon}=-\lim _{\Lambda \rightarrow \mathbb{R}^{n}} \frac{1}{|\Lambda|} \ln Z_{\Lambda}
$$

exists when $\Lambda \rightarrow \mathbb{R}^{n}$ in the sense that $d(x, C \Lambda)$ tends to infinity for all $x \in \mathbb{R}^{n}$. Moreover $\tilde{\varepsilon}(\lambda)$ is analytic ${ }^{17}$ in $\lambda$ for $|\lambda|<C^{-1} e^{-2 B-1}$ and

$$
\tilde{\varepsilon}(\lambda)=-\int_{0}^{\lambda} \frac{1}{\hat{\lambda}} \varrho^{1}(x s ; \tilde{\lambda}) d \tilde{\lambda} d v(s),
$$

where $\varrho^{1}(x s, \lambda)$ is the correlation function with one argument. For $\lambda<0$ and $d v$ a positive measure we have that $\varrho^{1}$ is positive which gives us that $\tilde{\varepsilon}$ is negative. Moreover in this case $\tilde{\varepsilon}$ exists also for all $\lambda<0$, decreases when $|\lambda|$ increases and is also concave in $\ln (-\lambda)$.

17 For all $|\lambda|<C^{-1} e^{-2 B-1}$ (and all $d v$ ) the expansions of $\varrho^{1}$ and $(-\tilde{\varepsilon})$ in powers of $\lambda$ are the Mayer series $\Sigma\left(n b_{n}\right)(-\lambda)^{n}$ resp. $\Sigma b_{n}(-\lambda)^{n}$ for the "density" respectively "pressure" of our "gas" in $\mathbb{R}^{n}$. Hence information on the expansion coefficients is readily available (see e.g. [15], p. 84-86).

Note that the well known virial expansion (of the pressure in powers of the density) corresponds in our case to an expansion of $-\tilde{\varepsilon}$ in powers of $\varrho^{1}$, expansion which can be obtained by inverting the expansion of $\varrho^{1}$ in powers of $\lambda$ in a neighborhood of $\lambda=0$ (which is possible since $\lim _{\lambda \rightarrow 0} \frac{\varrho^{1}}{i} \neq 0$ ). 
Proof. From the expansion of $Z_{A}$ (4.2) and the expansion of $\varrho_{A}^{1}(4.3)$ we find that

$$
\frac{d}{d \lambda} \ln Z_{\Lambda}=\frac{1}{\lambda} \int_{\Lambda} \varrho_{\Lambda}^{1}(x s ; \lambda) d v(s) d x
$$

From Lemma 4.1 we have that $\frac{1}{\lambda} \varrho_{A}^{1}(x s, \lambda)$ is uniformly bounded and analytic in $\lambda$ for $|\lambda|<C^{-1} e^{-2 B-1}-\delta$, for any $\delta>0$. Moreover $\left|\varrho_{\Lambda}^{1}(x, s ; \lambda)-\varrho^{1}(x, s ; \lambda)\right| \leqq C^{-1} \eta(d)$, and hence it follows that

$$
\frac{1}{|\Lambda|} \int_{\Lambda} d \nu(s) d x \int_{0}^{\lambda} \frac{1}{\lambda} \varrho_{\Lambda}^{1}(x, s ; \tilde{\lambda}) d \tilde{\lambda}
$$

converges uniformly for $|\lambda|<C^{-1} e^{-2 B-1}-\delta$ to

$$
\iint_{0}^{\lambda} \frac{1}{\lambda} \varrho^{1}(x, s ; \tilde{\lambda}) d v(s) d \tilde{\lambda},
$$

since $\varrho^{1}(x, s ; \tilde{\lambda})$ is independent of $x$. This proves that $\frac{1}{|\Lambda|} \ln Z_{\Lambda}$ converges as $\Lambda \rightarrow \mathbb{R}^{n}$ and that the limit- $\tilde{\varepsilon}$ is given by the formula of the Lemma. That $\varrho^{1}$ is positive for $d v \geqq 0$ and $\lambda \leqq 0$ follows from the fact that $\varrho_{A}^{1} \geqq 0$, which one sees from (4.3). The existence of $\tilde{\varepsilon}$ for all $\lambda<0$ in this case follows from the identification, possible in this case, of $Z_{A}$ with a grand canonical partition function for a system with stable and tempered interactions (see [15], p. 157).

The decrease of $\check{\varepsilon}$ as $|\lambda|$ increases follows from the increase of $Z_{\Lambda}$.

Remark. All the series expansions for the $\varrho^{r}\left(x_{1} s_{1}, \ldots, x_{r} s_{r}\right)$ and therefore also for $\tilde{\varepsilon}$ in powers of $\lambda$ can be explicitely obtained from (4.13) and are given by

$$
\varrho=-\lambda(1+\lambda \Pi K)^{-1} \alpha=-\lambda \sum_{n=0}^{\infty}(-\lambda)^{n}(\Pi K)^{n} \boldsymbol{\alpha} .
$$

\section{Removal of the Space Cut-off for the Imaginary Time Wightman Functions and the Vacuum Energy Density}

Let $\Lambda_{t, l}=[-t / 2, t / 2] \times\{\boldsymbol{x}|| \boldsymbol{x} \mid \leqq l\} \subset \mathbb{R}^{n}$ and set $Z_{t, l}=Z_{\Lambda_{t, l}}$ and $F_{t, l}$ $=F_{A_{t, l}}$ and $G_{t, l}=G_{\Lambda_{t, l}}$. It then follows from Lemma 3.3 that

$$
Z_{t, l}=\left(\Omega_{0}, e^{-t H_{l}} \Omega_{0}\right),
$$


with $H_{l}=H_{0}+\lambda \int_{|x| \leqq l} v\left(\varphi_{\varepsilon}(\boldsymbol{x})\right) d \boldsymbol{x}$. From (3.8) and (3.9) we have, for $h_{1}, \ldots, h_{k}$ with support in $\Lambda_{t, l}$, that

$$
\begin{gathered}
F_{t, l}^{k}\left(h_{1}, \ldots, h_{k}\right) \\
\left.=k ! \int_{t_{1} \leqq \ldots \leqq t_{k}} \ldots \Omega_{0}, e^{-\left(t_{1}+t / 2\right) H_{l}} \varphi\left(\boldsymbol{x}_{1}\right) e^{-\left(t_{2}-t_{1}\right) H_{l}} \varphi\left(\boldsymbol{x}_{2}\right) \ldots \varphi\left(\boldsymbol{x}_{k}\right) e^{-\left(t / 2-t_{k}\right) \boldsymbol{H}_{l}} \Omega_{0}\right) \\
\cdot h_{1}\left(t_{1}, \boldsymbol{x}_{1}\right) \ldots h_{k}\left(t_{k}, \boldsymbol{x}_{k}\right) \prod_{j=1}^{k} d t_{j} d \boldsymbol{x}_{j},
\end{gathered}
$$

and

$$
G_{t, l}^{k}=Z_{t, l}^{-1} F_{t, l}^{k} .
$$

By Lemma 3.4 the limit as $t \rightarrow+\infty$ of $G_{t, l}^{k}$ exists and is given by

$$
\begin{aligned}
& G_{l}^{k}\left(h_{1}, \ldots, h_{k}\right) \\
& =k ! \int_{t_{1} \leqq \cdots \leqq t_{k}}\left(\Omega_{l}, \varphi\left(\boldsymbol{x}_{1}\right) e^{-\left(t_{2}-t_{1}\right) \bar{H}_{l}} \ldots e^{-\left(t_{k}-t_{k-1}\right) \bar{H}_{l}} \varphi\left(\boldsymbol{x}_{k}\right) \Omega_{l}\right) \\
& \cdot h_{1}\left(t_{1}, \boldsymbol{x}_{1}\right) \ldots h_{k}\left(t_{k}, \boldsymbol{x}_{k}\right) \prod_{j=1}^{k} d t_{j} d \boldsymbol{x}_{j},
\end{aligned}
$$

where $\Omega_{l}$ is the unique normalized eigenvector with eigenvalue $E_{l}$ and $E_{l}$ is the infimum of the spectrum of $H_{l}$, and $\bar{H}_{l}=H_{l}-E_{l}$. The integration over $d \boldsymbol{x}_{j}$ in (5.2) and (5.4) is to be understood in the sense of distributions. After integrating with respect to $\prod_{j=1}^{k} d \boldsymbol{x}_{j}$ in (5.2) and (5.4), the result is a function of $t_{1}, \ldots, t_{k}$ that is translation invariant, continuous in $t_{1}<\cdots<t_{k}$ and integrable over $t_{1} \leqq \cdots \leqq t_{k}$. This follows from the proof of Lemma 3.4. We see from (5.4) that $G_{l}^{k}\left(h_{1}, \ldots, h_{k}\right)$ are the imaginary time Wightman functions (also called Schwinger functions) for the space cut-off interaction.

Theorem 5.1. Let $|\lambda|<C^{-1} e^{-2 B-1}$ and $h_{1}, \ldots, h_{k}$ be in $C_{0}^{\infty}$. Then the $G_{l}^{k}\left(h_{1}, \ldots, h_{k}\right)$ converge as $l \rightarrow \infty$ to $G^{k}\left(h_{1}, \ldots, h_{k}\right)$, where $G^{k}\left(h_{1}, \ldots, h_{k}\right)$ are translation invariant in $t$ and $\boldsymbol{x}$ and given by

$$
\begin{gathered}
G^{k}\left(h_{1}, \ldots, h_{k}\right)=G_{0}^{k}\left(h_{1}, \ldots, h_{k}\right)+(i)^{k} \sum_{r=1}^{k} \frac{1}{r !} \sum_{\substack{p+q=k \\
q \geqq r, p \geqq 0}} \frac{(i)^{p}}{p !} \\
\sum_{\sigma \in S_{k}} G_{0}^{k}\left(h_{\sigma(1)}, \ldots, h_{\sigma(p)}\right) \sum_{\substack{l_{1}+\cdots+l_{r}=q \\
l_{l} \geqq 1}} \frac{1}{l_{1} ! \ldots l_{r} !} \\
\cdot \int \cdots \int \prod_{i=1}^{r}\left[s_{i}^{l_{i}} \prod_{j=1}^{l_{l}} h_{\sigma\left(p+l_{1}+\cdots+l_{t}-j+1\right)}^{\varepsilon}\left(x_{i}\right)\right] \\
\cdot \varrho^{r}\left(x_{1} s_{1}, \ldots, x_{r} s_{r}\right) \prod_{j=1}^{r} d v\left(s_{j}\right) d x_{J} .
\end{gathered}
$$


$S_{k}$ is the set of permutations of $1, \ldots, k$ and the $G_{0}^{k}\left(h_{1}, \ldots, h_{k}\right)$ are the imaginary time free Wightman functions: $G_{0}^{k}\left(h_{1}, \ldots, h_{k}\right)=E\left(\Phi\left(h_{1}\right) \ldots \Phi\left(h_{k}\right)\right)$ $=\frac{1}{2^{p} p !} \sum_{\sigma \in S_{k}}\left(h_{\sigma(1)}, h_{\sigma(2)}\right)_{-1} \ldots\left(h_{\sigma(2 p-1)}, h_{\sigma(2 p)}\right)_{-1}$ for $k=2 p$ and zero for $k$ odd. $\varrho^{r}\left(x_{1} s_{1}, \ldots, x_{r} s_{r}\right)$ is the infinite volume correlation function of Lemma 4.1, and $h_{i}^{\varepsilon}(x)=\int G_{\varepsilon}(x-y) h_{i}(y) d y$ and $G_{\varepsilon}(x)$ is the $G_{\varepsilon}(x)$ of Section 4, which is given by

$$
G_{\varepsilon}(x)=\int \frac{e^{i p x} \tilde{\chi}_{\varepsilon}^{2}(\boldsymbol{p})}{p^{2}+m^{2}} d p,
$$

with $\tilde{\chi}_{\varepsilon}(\boldsymbol{p})=\int e^{i \boldsymbol{p} \boldsymbol{x}} \chi_{\varepsilon}(\boldsymbol{x}) d \boldsymbol{x}$.

Proof. It follows from (4.4) and the fact that $G_{\Lambda}\left(\sum_{i=1}^{k} t_{i} h_{i}\right)$ is analytic in $t_{1}, \ldots, t_{k}$ that the formula (5.5) with $G_{A}^{k}\left(h_{1}, \ldots, h_{k}\right)$ instead of $G^{k}\left(h_{1}, \ldots, h_{k}\right)$ and with $\varrho_{\Lambda}^{r}\left(x_{1} s_{1}, \ldots, x_{r} s_{r}\right)$ instead of $\varrho^{r}\left(x_{1} s_{1}, \ldots, x_{r} s_{r}\right)$ holds. Choosing now $\Lambda=\Lambda_{t, l}$ we have by (5.4) that $G_{t, l}^{k}\left(h_{1}, \ldots, h_{k}\right)$ converges to the limit $G_{l}^{k}\left(h_{1}, \ldots, h_{k}\right)$ as $t \rightarrow \infty$. On the other hand by Lemma $4.1 \varrho_{\Lambda_{t, l}}^{r}\left(x_{1} s_{1} \ldots x_{r} s_{r}\right)$ is uniformly bounded in $x_{1}, \ldots, x_{r}, t, l$ and tends to a limit $\varrho^{r}\left(x_{1} s_{1}, \ldots, x_{r} s_{r}\right)$ uniformly on compacts as $t$ and $l$ tend to infinity. Since $h_{i}^{\varepsilon}(x) i=1, \ldots, k$ are all bounded integrable functions we get by dominated convergence from (5.5), with $G_{t, l}^{k}=G_{A_{t, l}}^{k}$ and $\varrho_{A_{t, l}}^{k}$ instead of $G^{k}$ and $\varrho^{r}$, that $G_{t, l}^{k}\left(h_{1}, \ldots, h_{k}\right)$ converges to the limit $G^{k}\left(h_{1}, \ldots, h_{k}\right)$, given by (5.5), as $t$ and $l$ tend to infinity.

Consider now the inequality

$$
\begin{aligned}
& \left|G_{l}^{k}\left(h_{1}, \ldots, h_{k}\right)-G^{k}\left(h_{1}, \ldots, h_{k}\right)\right| \\
& \leqq\left|G_{l}^{k}\left(h_{1}, \ldots, h_{k}\right)-G_{t, l}^{k}\left(h_{1}, \ldots, h_{k}\right)\right|+\left|G_{t, l}^{k}\left(h_{1}, \ldots, h_{k}\right)-G^{k}\left(h_{1}, \ldots, h_{k}\right)\right| .
\end{aligned}
$$

Choose $\varepsilon>0$; then there exists a $N_{\varepsilon}$ such that for any $t \geqq N_{\varepsilon}$ and any $l \geqq N_{\varepsilon}$ the last term is smaller than $\varepsilon / 2$. Choose an $l \geqq T_{\varepsilon}$. Then for this value of $l$ we may choose a $t \geqq N_{\varepsilon}$ and large enough so that the first term is smaller than $\varepsilon / 2$. Then for $l \geqq T_{\varepsilon}$ we get $\left|G_{l}^{k}\left(h_{1}, \ldots, h_{k}\right)-G^{k}\left(h_{1}, \ldots, h_{k}\right)\right| \leqq \varepsilon$. This proves the theorem.

Theorem 5.2. Let $|\lambda|<C^{-1} e^{-2 B-1}$, and let $h_{1}, \ldots, h_{k}, g_{1}, \ldots, g_{l}$ be in $C_{0}^{\infty}\left(\mathbb{R}^{n}\right)$.

Let $g_{i}^{a}(x)=g_{i}(x-a)$ for $a \in \mathbb{R}^{n}$. Then we have the following cluster properties:

$$
G^{k+l}\left(h_{1}, \ldots, h_{k}, g_{1}^{a}, \ldots, g_{l}^{a}\right) \rightarrow G^{k}\left(h_{1}, \ldots, h_{k}\right) G^{l}\left(g_{1}, \ldots, g_{l}\right)
$$

as $|a| \rightarrow \infty$. 
Proof. It follows from (5.5) that for any $h \in C_{0}^{\infty}\left(\mathbb{R}^{n}\right)$

$$
G(h)=1+\sum_{k=1}^{\infty} \frac{(i)^{k}}{k !} G^{k}(h, \ldots, h) \text { is defined }
$$

and the series is absolutely convergent. Remembering that (5.5) was obtained by means of (4.4), we get

$$
\begin{aligned}
G(h)= & e^{-\frac{1}{2}(h, h)-1}\left[1+\sum_{r=1}^{\infty} \frac{1}{r !} \int \cdots \int \prod_{j=1}^{r}\left(e^{-s_{j} h^{\varepsilon}\left(x_{j}\right)}-1\right)\right. \\
& \left.\cdot \varrho^{r}\left(x_{1} s_{1}, \ldots, x_{r} s_{r}\right) \prod_{j=1}^{r} d v\left(s_{j}\right) d x_{j}\right] .
\end{aligned}
$$

Therefore

$$
\begin{aligned}
G\left(h^{a}+g^{-a}\right)=e^{-\frac{1}{2}(h, h)-1} \cdot e^{-\frac{1}{2}(g, g)-1} e^{-\left(h^{a}, g^{-a}\right)-1} \\
{\left[1+\sum_{r=1}^{\infty} \frac{1}{r !} \int \cdots \int \prod_{j=1}^{r}\left(e^{-s_{j} h^{\varepsilon}\left(x_{j}-a\right)-s_{j} g^{\varepsilon}\left(x_{j}+a\right)}-1\right)\right.} \\
\left.\cdot \varrho^{r}\left(x_{1} s_{1}, \ldots, x_{r} s_{r}\right) \prod_{j=1}^{r} d v\left(s_{j}\right) d x_{j}\right] .
\end{aligned}
$$

We observe that $\left(h^{a}, g^{-a}\right)_{-1} \rightarrow 0$ as $|a| \rightarrow \infty$. By writing each of the integrals over $x_{i}$ as the sum of the integrals over $x_{i} \cdot a \leqq 0$ and $x_{i} \cdot a \geqq 0$, we get that the $r$ 'th term of the series above is equal to

$$
\begin{aligned}
& \frac{1}{r !} \sum_{s=0}^{r}\left(\begin{array}{l}
r \\
S
\end{array}\right) \int_{x_{j} \cdot a \geqq 0} \cdots \int_{y_{j} \cdot a \leqq 0} \cdot \int_{j=1} \cdots \prod_{j} \prod_{j}\left(e^{-s_{J} h^{\varepsilon}\left(x_{j}-a\right)-s_{J} g^{\varepsilon}\left(x_{J}+a\right)}-1\right) \\
& \cdot \prod_{j=1}^{r-s}\left(e^{-t_{J} h^{\varepsilon}\left(y_{j}-a\right)-t_{J} g^{\varepsilon}\left(y_{J}+a\right)}-1\right) \varrho^{r}\left(x_{1} s_{1} \ldots x_{s} s_{s}, y_{1} t_{1} \ldots y_{r-s} t_{r-s}\right) \\
& \cdot \prod_{j=1}^{s} d v\left(s_{j}\right) d x_{j} \cdot \prod_{j=1}^{r-s} d v\left(t_{j}\right) d y_{j} .
\end{aligned}
$$

From the definition of $h^{\varepsilon}(x)=\int G_{\varepsilon}(x-y) h(y) d y$ we get that $\left|h^{\varepsilon}(x)\right|$ $\leqq C e^{-m|x|}$, from which we obtain that

$$
\left|h^{\varepsilon}(y-a)\right| \leqq C e^{-\frac{m}{2}|a|} \cdot e^{-\frac{m}{2}|y|} \text { for } y \cdot a \leqq 0
$$

and similarly

$$
\left|g^{\varepsilon}(x+a)\right| \leqq C e^{-\frac{m}{2}|a|} \cdot e^{-\frac{m}{2}|x|} \text { for } \quad x \cdot a \geqq 0 .
$$


By the substitution $x_{j} \rightarrow x_{j}+a$ and $y_{j} \rightarrow y_{j}-a$ we get

$$
\begin{aligned}
& \frac{1}{r !} \sum_{s=0}^{r}\left(\begin{array}{l}
r \\
s
\end{array}\right) \int_{x_{j} \cdot a \geqq-a^{2}} \cdots \int_{y_{j} \cdot a \leqq a^{2}} \cdots \prod_{j=1}^{s}\left(e^{-s_{j} h^{\varepsilon}\left(x_{j}\right)-s_{,} g^{\varepsilon}\left(x_{J}+2 a\right)}-1\right) \\
& \cdot \prod_{j=1}^{r-s}\left(e^{-t_{j} h^{\varepsilon}\left(y_{j}-2 a\right)-t_{j} g^{\varepsilon}\left(y_{j}\right)}-1\right) \varrho_{a}^{r}\left(x_{1} s_{1} \ldots x_{s} s_{s}, y_{1} t_{1} \ldots y_{r-s} t_{r-s}\right)
\end{aligned}
$$

$\cdot \Pi d v\left(s_{j}\right) d x_{j} \Pi d v\left(t_{j}\right) d y_{j}$

where $\varrho_{a}^{r}\left(x_{1}, \ldots, x_{s}, y_{1}, \ldots, y_{r-s}\right)=\varrho^{r}\left(x_{1}+a, \ldots, x_{s}+a, y_{1}-a, \ldots, y_{r-s}-a\right)$. Let $F_{a}(x, s)$ be any measurable function uniformly bounded in $a$; then

$$
\begin{aligned}
& \int_{x \cdot a \geqq-a^{2}}\left(e^{-s h^{\varepsilon}(x)-s g^{\varepsilon}(x+2 a)}-1\right) F_{a}(x, s) d x d v(s) \\
& -\int_{x \cdot a \geqq-a^{2}}\left(e^{-s h^{\varepsilon}(x)}-1\right) F_{a}(x, s) d x d v(s)
\end{aligned}
$$

converges to zero when $|a| \rightarrow \infty$, because the absolute value of $(5.10)$ is bounded by

$$
\begin{aligned}
& \quad \int_{x \geqq-a^{2}} e^{-s h^{\varepsilon}(x)}\left|e^{-s g^{\varepsilon}(x+2 a)}-1\right|\left|F_{a}(x, s)\right| d x d v(s) \\
& \leqq A \int_{x \cdot a \geqq-a^{2}}\left|e^{-s g^{\varepsilon}(x+2 a)}-1\right| d x d v(s) \\
& \leqq B \int_{x \cdot a \geqq-a^{2}}\left|g^{\varepsilon}(x+2 a)\right| d x=B \int_{x \cdot a \geqq 0}\left|g^{\varepsilon}(x+a)\right| d x \\
& \leqq B \cdot C e^{-\frac{m}{2}|a|} \int e^{-\frac{m}{2}|x|} d x .
\end{aligned}
$$

Therefore for any $\varepsilon>0$ there exists an $R_{\varepsilon}$ such that, for $|a|>R_{\varepsilon},(5.9)$ will differ from (5.11) by an amount smaller than $\varepsilon / 2$ :

$$
\begin{aligned}
& \frac{1}{r !} \sum_{s=0}^{r} \underset{x_{j} \cdot a \geqq-a^{2}}{\int \cdots \int_{y_{j} \cdot a \leqq a^{2}}} \cdot \prod_{j=1}^{s}\left(e^{-s_{j} h^{\varepsilon}\left(x_{j}\right)}-1\right) \prod_{j=1}^{r-s}\left(e^{-t_{J} g^{\varepsilon}\left(y_{j}\right)}-1\right) \\
& \cdot Q_{a}^{r}\left(x_{1} s_{1}, \ldots, x_{s} s_{s}, y_{1} t_{1}, \ldots, y_{r-s} t_{r-s}\right) \prod_{j=1}^{s} d v\left(s_{j}\right) d x_{j} \prod_{j=1}^{r-s} d v\left(t_{j}\right) d y_{j} .
\end{aligned}
$$

By dominated convergence and Lemma 4.2 we have that (5.11) converges to (5.12) as $|a| \rightarrow \infty$ :

$$
\begin{aligned}
& \frac{1}{r !} \sum_{s=0}^{r}\left(\begin{array}{l}
r \\
s
\end{array}\right) \int \cdots \int \prod_{j=1}^{s}\left(e^{-s_{J} h^{\varepsilon}\left(x_{J}\right)}-1\right) \varrho^{s}\left(x_{1} s_{1}, \ldots, x_{s} s_{s}\right) \prod_{j=1}^{s} d v\left(s_{j}\right) d x_{j} \\
& \quad \cdot \int \cdots \int \prod_{j=1}^{r-s}\left(e^{-t_{j} g^{\varepsilon}\left(y_{j}\right)}-1\right) \varrho^{r-s}\left(y_{1} t_{1}, \ldots, y_{r-s} t_{r-s}\right) \prod_{j=1}^{r-s} d v\left(t_{j}\right) d y_{j} .
\end{aligned}
$$


From (5.7) and the translation invariance we now get that

$$
G\left(h+g^{a}\right) \rightarrow G(h) G(g) \quad \text { as } \quad|a| \rightarrow \infty .
$$

Since $G\left(\sum_{i} t_{i} h_{i}+\sum_{j} s_{j} g_{j}^{a}\right)$ is analytic in $t$ and $s$ and converges to $G\left(\sum_{l} t_{i} h_{\imath}\right)$ - $G\left(\sum_{j} s_{j} g_{j}\right)$, we have only to use that the convergence of analytic functions implies the convergence of the coefficients of their powerseries to prove that $G^{k+l}\left(h_{1}, \ldots, h_{k}, g_{1}^{a}, \ldots, g_{l}^{a}\right)$ converges to $G^{k}\left(h_{1}, \ldots, h_{k}\right) G^{l}\left(g_{1}, \ldots, g_{l}\right)$.

Theorem 5.3. Set

$$
G^{k}\left(h_{1}, \ldots, h_{k}\right)=\int \cdots \int G^{k}\left(x_{1}, \ldots, x_{k}\right) h_{1}\left(x_{1}\right), \ldots, h_{k}\left(x_{k}\right) d x_{1}, \ldots, d x_{k},
$$

then $G^{k}\left(x_{1}, \ldots, x_{k}\right)$ is locally integrable and continuous for $x_{i} \neq x_{j}$, for all $i \neq j$. The singularities at $x_{i}=x_{j}$ are of the same form as the singularities of $G_{0}^{k}\left(x_{1}, \ldots, x_{j}\right)$. Moreover the $G^{k}\left(x_{1}, \ldots, x_{k}\right)$ are translation invariant and, for $\chi_{\varepsilon}(\boldsymbol{x})$ rotational invariant, they are also invariant under rotations in $\mathbb{R}^{n-1}$. The $G^{k}\left(x_{1}, \ldots, x_{k}\right)$ depend analytically on $\lambda$ for $|\lambda|<C^{-1} e^{-2 B-118}$.

Proof. This follows from (5.5) and the analyticity of the $\varrho^{r}\left(x_{1}, s_{1}, \ldots, x_{r} s_{r}\right)$ as proved in Lemma 4.1.

Theorem 5.4. For all $|\lambda|<C^{-1} e^{-2 B-1}$ we have that the vacuum energy density

$$
\tilde{\varepsilon}=\lim _{l \rightarrow \infty}\left|B_{l}\right|^{-1} E_{l}
$$

exists, where $\left|B_{l}\right|$ is the volume of the $n-1$-dimensional ball of radius $l$. Moreover this limit is equal to the $\tilde{\varepsilon}$ of Lemma 4.3 and is therefore analytic in $\lambda$ for all $|\lambda|<C^{-1} e^{-2 B-1}$ and its power series is given in terms of the powerseries for $\varrho^{1}$ by

$$
\tilde{\varepsilon}(\lambda)=-\int_{0}^{\lambda} \frac{1}{\lambda} \varrho^{1}(x, s ; \tilde{\lambda}) d \tilde{\lambda} d v(s) .
$$

The power series for all $Q^{r}$, hence also for $\tilde{\varepsilon}$, are explicitely given by $(4.14)^{19}$.

$\tilde{\varepsilon}(\lambda)$ is a concave function of $\lambda$.

For $d v$ a positive measure and all $\lambda<0$ (not necessarily $>-C^{-1} e^{-2 B-1}$ ) the limit $\tilde{\varepsilon}$ exists, is negative, decreasing for $|\lambda|$ increasing, concave in $\lambda$ and $\ln (-\lambda)$.

18 The coefficients in the expansion of $G^{k}\left(x_{1}, \ldots, x_{k}\right)$ in powers of $\lambda$ can be obtained from those of the expansions of the $\varrho$ 's in powers of $\lambda$, using (5.5). The latter are known and given by (4.14) (see also footnote 17).

19 The expansion for $(-\tilde{\varepsilon}(\lambda))$ is the Mayer power series expansion $\Sigma b_{n}(-\lambda)^{n}$ for the "pressure" of our gas as a function of $(-\lambda)$. Hence the coefficient are the quantities $b_{n}$ of footnote 17, which can be computed explicitely. 
Proof. By Lemma $4.3\left|\Lambda_{t, l}\right|^{-1} \ln Z_{t, l}$ converges to $\tilde{\varepsilon}$ as $t$ and $l$ tend to infinity. We observe that $\left|\Lambda_{t, l}\right|=t \cdot\left|B_{l}\right|$. By (5.1) $Z_{t, l}=\left(\Omega_{0}, e^{-t H_{l}} \Omega_{0}\right)$. $E_{l}$ is a simple lowest eigenvalue of $H_{l}$ so that $\frac{1}{t} \ln \left(\Omega_{0}, e^{-t H_{l}} \Omega_{0}\right) \rightarrow-E_{l}$ as $t \rightarrow \infty$. Therefore

$$
\left|\Lambda_{t, l}\right|^{-1} \ln Z_{t, l} \rightarrow-\left|B_{l}\right|^{-1} \cdot E_{l}
$$

as $t \rightarrow \infty$. Since $\left|\Lambda_{t, l}\right|^{-1} \ln Z_{t, l}$ converges to $\tilde{\varepsilon}$ for $t$ and $l$ tending to infinity it now follows that $\left|B_{l}\right|^{-1} E_{l}$ converges as $l \rightarrow \infty$ to the $\tilde{\varepsilon}$ of Lemma 4.3. That $\tilde{\varepsilon}$ is concave in $\lambda$ follows from $\tilde{\varepsilon}$ being the limit of $-\left|B_{l}\right|^{-1} E_{l}$ and the fact that $E_{l}$, being the lowest eigenvalue of $H_{l}=H_{0}+\lambda V_{l}$, is concave in $\lambda$. The rest of the theorem is contained in Lemma 4.3.

\section{The Vacuum, the Interacting Fields and the Wightman Functions}

Let $\alpha_{t}^{l}(A)=e^{-i t H_{l}} A e^{i t H_{l}}$, for any bounded operator $A$ on $\mathscr{F}$, and let $\alpha_{t}^{0}$ be the corresponding one parameter group of $C^{*}$-automorphisms defined with $H_{0}$ instead of $H_{l}$. Let $\mathscr{A}_{0}^{T}(\Omega)$, for any open domain $\Omega$ in $\mathbb{R}^{n-1}$, be the $W^{*}$-algebra generated by all $\alpha_{t}^{0}\left(e^{i \varphi(f)}\right)$, for all $t \in[-T, T]$ and all $f \in \mathscr{H}_{n-1}^{-\frac{1}{1}}$ with compact support in $\Omega$, where $\varphi(f)=\int \varphi(\boldsymbol{x}) f(\boldsymbol{x}) d \boldsymbol{x}$, with $\varphi(x)$ given by (2.1). Let $\mathscr{A}_{0}$ be the smallest $C^{*}$-algebra containing all $\mathscr{A}_{0}^{T}(\Omega)$.

Theorem 6.1. $\alpha_{t}^{l}$ as well as $\alpha_{t}^{0}$ are one parameter groups of $C^{*}$-automorphisms of $\mathscr{A}_{0}$. Moreover $\alpha_{t}^{l}$ converges strongly on $\mathscr{A}_{0}$ to a one parameter group of automorphisms $\alpha_{t}$ of $\mathscr{A}_{0}$ as $l \rightarrow \infty$. We also have that $\alpha_{-t}^{0} \alpha_{t}^{l}$ and $\alpha_{t}^{l} \alpha_{-t}^{0}$ converge strongly to $\alpha_{-t}^{0} \alpha_{t}$ and $\alpha_{t} \alpha_{-t}^{0}$, uniformly on an open interval containing $t=0$.

The proof of this theorem is entirely similar to the proof of the corresponding theorem in Ref. [6d]. The only difference is that we do not assume that $\alpha_{t}^{0}$ is strongly continuous. As mentioned in [6d], p. 31, this can be overcome by taking, as we have done, the local $W^{*}$-closure in forming the algebra $\mathscr{A}_{0}$.

The conclusions of Theorem 6.1 are, however, weaker than those of the corresponding theorem in [6d], in as much as we can not say that $\alpha_{t}$ is strongly continuous ${ }^{20}$.

Let now $Z_{A}, F_{A}$ and $f_{x}$ be as in Section 4 , where we have also defined $\Phi_{\varepsilon}(x)=\Phi\left(f_{x}\right), \quad \Phi=\Phi_{\mathscr{H}_{n}^{-1}}$ being the generalized Gaussian stochastic process indexed by the Sobolev space $\mathscr{H}_{n}^{-1}$. Then

$(-\lambda)^{k} Z_{\Lambda}^{-1} F_{\Lambda}\left(\sum_{j=1}^{k} s_{j} f_{x_{j}}\right)=(-\lambda)^{k} Z_{\Lambda}^{-1} E\left(e^{i \sum_{j=1}^{k} s_{j} \Phi_{\varepsilon}\left(x_{j}\right)} e^{-\lambda \int_{\Lambda} v\left(\Phi_{\varepsilon}(x) d x\right)}\right)$,

${ }^{20}$ For additional results on the $\alpha_{t}^{l}$ see [6b], Lemma 4. 
where $E$ is the expectation in the probability space of the generalized process $\Phi$. If we now expand with respect to $\lambda$ we get that this is equal to

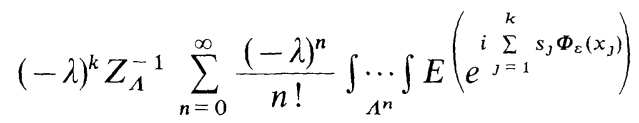

$$
\begin{aligned}
& \cdot v\left(\Phi_{\varepsilon}\left(x_{k+1}\right)\right) \ldots v\left(\Phi_{\varepsilon}\left(x_{k+n}\right)\right) \prod_{j=k+1}^{k+n} d x_{j} \\
& =Z_{\Lambda}^{-1} \sum_{n=0}^{\infty} \frac{(-\lambda)^{n+k}}{n !} \int \cdots \int E\left(e^{n} e^{i \sum_{j=1}^{k+n} s_{j} \Phi_{\varepsilon}\left(x_{j}\right)}\right) \prod_{j=k+1}^{k+n} d v\left(s_{j}\right) d x_{j} \\
& =Z_{\Lambda}^{-1} \sum_{n=0}^{\infty} \frac{(-\lambda)^{n+k}}{n !} \iint_{\Lambda^{n}} \int e^{-\frac{1}{2} \sum_{i, j=1}^{n+k} s_{i} s_{j} G_{\varepsilon}\left(x_{2}-x_{j}\right)} \prod_{j=k+1}^{k+n} d v\left(s_{j}\right) d x_{j},
\end{aligned}
$$

which by (4.3) is equal to $\varrho_{A}^{k}\left(x_{1} s_{1}, \ldots, x_{k} s_{k}\right)$.

This proves the formula:

$$
\begin{aligned}
& \varrho_{\Lambda}^{k}\left(x_{1} s_{1}, \ldots, x_{k} s_{k}\right) \\
& =(-\lambda)^{k} E\left(e^{-\lambda \int_{\Lambda} v\left(\Phi_{\varepsilon}(x)\right) d x}\right)-1 E\left(e^{i \sum_{j=1}^{k} s_{J} \Phi_{\varepsilon}\left(x_{J}\right)} e^{-\lambda \int_{\Lambda} v\left(\Phi_{\ell}(x)\right) d x}\right) .
\end{aligned}
$$

Choose now $\Lambda=\Lambda_{a, l}=\left\{\left(x_{0}, \boldsymbol{x}\right) ;\left|x_{0}\right| \leqq a,|\boldsymbol{x}| \leqq l\right\}$. Then by Lemma 3.2, for $-a \leqq t_{1} \leqq \cdots \leqq t_{k} \leqq a$, where $t_{i}=\left(x_{i}\right)_{0}, i=1, \ldots, k$ :

$$
E\left(e^{-\lambda \int v\left(\Phi_{t}(x)\right) d x}\right)=\left(\Omega_{0}, e^{-2 a H_{l}} \Omega_{0}\right),
$$

and, with $\varphi_{\varepsilon}(\boldsymbol{x})$ given by (2.2),

$$
\begin{aligned}
& E\left(e^{\left(i \sum_{j=1}^{k} s_{J} \Phi_{\varepsilon}\left(x_{j}\right)\right.} e^{-\lambda \int_{\Lambda} v\left(\Phi_{\varepsilon}(x)\right) d x}\right) \\
& \quad=\left(\Omega_{0}, e^{-\left(t_{1}+a\right) H_{l}} e^{i s_{1} \varphi_{\varepsilon}\left(\boldsymbol{x}_{1}\right)} e^{-\left(t_{2}-t_{1}\right) H_{l}} \ldots e^{i s_{k} \varphi_{\varepsilon}\left(\boldsymbol{x}_{k}\right)} e^{-\left(a-t_{k}\right) H_{l}} \Omega_{0}\right) .
\end{aligned}
$$

Since $E_{l}$ is a simple isolated lowest eigenvalue, the limit as $a \rightarrow \infty$ of the expression obtained dividing (6.4) by (6.3) exists and is equal to

$$
\left(\Omega_{l}, e^{i s_{1} \varphi_{\varepsilon}\left(\boldsymbol{x}_{1}\right)} e^{-\left(t_{2}-t_{1}\right) \bar{H}_{l}} \ldots e^{-\left(t_{k}-t_{k-1}\right) \bar{H}_{l}} e^{i s_{k} \varphi_{\varepsilon}\left(\boldsymbol{x}_{k}\right)} \Omega_{l}\right) .
$$

We have the following theorem:

Theorem 6.2. The functions $\varrho_{A_{a, l}}^{k}\left(x_{1} s_{1}, \ldots, x_{k} s_{k}\right)$ converge pointwise as $a \rightarrow \infty$ to the finite volume correlation functions $\varrho_{l}^{k}\left(x_{1} s_{1}, \ldots, x_{k} s_{k}\right)$, where, for $t_{i}=\left(x_{i}\right)_{0}$ and $t_{1} \leqq \cdots \leqq t_{k}$ :

$$
\begin{aligned}
& \varrho_{l}^{k}\left(x_{1} s_{1}, \ldots, x_{k} s_{k}\right) \\
& \quad=(-\lambda)^{k}\left(\Omega_{l}, e^{i s_{1} \varphi_{\varepsilon}\left(\boldsymbol{x}_{1}\right)} e^{-\left(t_{2}-t_{1}\right) \bar{H}_{l}} \ldots e^{-\left(t_{k}-t_{k-1}\right) \bar{H}_{l}} e^{i s_{k} \varphi_{\varepsilon}\left(\boldsymbol{x}_{k}\right)} \Omega_{l}\right),
\end{aligned}
$$

with $\bar{H}_{l}=H_{l}-E_{l}$. 
Moreover $G_{l}^{k}\left(h_{1}, \ldots, h_{k}\right)$ is given in terms of $\varrho_{l}^{k}$ by (5.5), where $\varrho_{l}^{k}$ is substituted for $Q^{k}$.

Proof. All but the moreover part is proven above, and the proof of the moreover part follows from the proof of Theorem 5.1.

From Theorem 6.2 it follows that $\varrho_{l}^{k}$ is analytic in $\xi_{1}=t_{2}-t_{1}, \ldots, \xi_{k-1}$ $=t_{k}-t_{k-1}$ for $\operatorname{Re} \xi_{i}>0, i=1, \ldots, k-1$, and uniformly bounded for $\operatorname{Re} \xi_{i} \geqq 0$. As in the proof of Theorem 5.1 we have that, for $|\lambda|<C^{-1} e^{-2 B-1}$ and real $t_{1}, \ldots, t_{k}, \varrho_{l}^{k}\left(x_{1} s_{1}, \ldots, x_{k} s_{k}\right)$ converges, uniformly on compacts, as $l \rightarrow \infty$, to $\varrho^{k}\left(x_{1} s_{1}, \ldots, x_{k} s_{k}\right)$. By the analyticity and uniform boundedness, for real $\lambda$ with $|\lambda|<C^{-1} e^{-2 B-1}$, in the region $\operatorname{Re} \xi_{l} \geqq 0$, $i=1, \ldots, k-1$, this implies that $\varrho_{l}^{k}\left(x_{1} s_{1}, \ldots, x_{k} s_{k}\right)$ converges for $\operatorname{Re} \xi_{1}>0$ pointwise to a function analytic in $\operatorname{Re} \xi_{i}>0, i=1, \ldots, k-1$, which is the analytic continuation of $\varrho^{k}\left(x_{1} s_{1}, \ldots, x_{k} s_{k}\right)$. Moreover the boundary values, i.e. the values on the set where all $t_{i}$ are purely imaginary, converge almost everywhere. This gives that

$$
\begin{aligned}
& \sigma_{l}^{k}\left(x_{1} s_{1}, \ldots, x_{k} s_{k}\right) \\
& \quad=(-i \lambda)^{k}\left(\Omega_{l}, e^{i s_{1} \varphi_{\varepsilon}\left(\boldsymbol{x}_{1}\right)} e^{-i\left(t_{2}-t_{1}\right) \bar{H}_{l}} \ldots e^{-l\left(t_{k}-t_{k}-1\right) \bar{H}_{l}} e^{i s_{k} \varphi_{\varepsilon}\left(\boldsymbol{x}_{k}\right)} \Omega_{l}\right)
\end{aligned}
$$

converges almost everywhere in the $t^{\prime}$ 's as $l \rightarrow \infty$, for all real $\lambda$ satisfying $|\lambda|<C^{-1} e^{-2 B-1}$.

Consider now the imaginary time Wightman functions $G^{k}\left(x_{1}, \ldots, x_{k}\right)$, given by the relation

$$
G^{k}\left(h_{1}, \ldots, h_{k}\right)=\int G^{k}\left(x_{1}, \ldots, x_{k}\right) h_{1}\left(x_{1}\right) \ldots h_{k}\left(x_{k}\right) d x_{1} \ldots d x_{k} .
$$

We then have:

Theorem 6.3. For real $\lambda$ with $|\lambda|<C^{-1} e^{-2 B-1}$ the imaginary time Wightman functions $G^{k}\left(x_{1}, \ldots, x_{k}\right)$ are analytic functions of all the variables $\xi_{1}=t_{2}-t_{1}, \ldots, \xi_{k-1}=t_{k}-t_{k-1}$ in the domain $\left\{\operatorname{Re} \xi_{i}>0, i=1, \ldots, k-1\right\}$, where $t_{i}=\left(x_{i}\right)_{0}$ for $i=1, \ldots, k$. Their boundary values on the imaginary axis, $W^{k}\left(x_{1}, \ldots, x_{k}\right)$ are the Wightman functions. $W^{k}\left(x_{1}, \ldots, x_{k}\right)$ satisfy the positive definiteness conditions for Wightman functions and are translation invariant in space and time. Moreover they are rotation invariant in space if $\chi_{\varepsilon}(\boldsymbol{x})$ is chosen rotation invariant.

Proof. From what is said before the theorem, we know that $\varrho^{k}\left(x_{1} s_{1}, \ldots, x_{k} s_{k}\right)$ is analytic and uniformly bounded for $\operatorname{Re} \xi_{i}>0$, $i=1, \ldots, k-1$. It follows then from (5.5) and the fact that $G_{\varepsilon}(t, x)$ is analytic for $\operatorname{Re} t>0$, that the $G^{k}\left(x_{1}, \ldots, x_{k}\right)$ are analytic for $\operatorname{Re} \xi_{i}>0$. Their boundary values $W^{k}\left(x_{1}, \ldots, x_{k}\right)$ for $\operatorname{Re} \xi_{i}=0, i=1, \ldots, k-1$ satisfy the positive definiteness conditions because they are limits in the 
sense of distributions ${ }^{21}$ of the finite volume Wightman functions, which are themselves boundary values of the functions $G_{l}^{k}\left(x_{1}, \ldots, x_{k}\right)$, analytic in $\operatorname{Re} \xi_{l}>0, i=1, \ldots, k-1$, satisfying for $t_{i}=\left(x_{i}\right)_{0}$ and $t_{1} \leqq \cdots \leqq t_{k}$

$$
G_{l}^{k}\left(x_{1}, \ldots, x_{k}\right)=\left(\Omega_{l}, \varphi\left(x_{1}\right) e^{-\left(t_{2}-t_{1}\right) \bar{H}_{l}} \ldots e^{-\left(t_{k}-t_{k}-1\right) \bar{H}_{l}} \varphi\left(\boldsymbol{x}_{k}\right) \Omega_{l}\right)
$$

and converging as $l \rightarrow \infty$ to $G^{k}\left(x_{1}, \ldots, x_{k}\right)$. The invariance of the $W^{k}\left(x_{1}, \ldots, x_{k}\right)$ follows from the corresponding invariance of the $G^{k}\left(x_{1}, \ldots, x_{k}\right)$, which was proven in Theorem 5.3.

Since the infinite volume Wightman functions $W^{k}\left(x_{1}, \ldots, x_{k}\right)$ satisfy the positive definiteness conditions, we can construct a Hilbert space $\mathscr{H}$ with a cyclic vector $\Omega$ in the usual fashion ${ }^{22}$, such that $\varphi(f)$ for $f$ smooth $^{23}$ are symmetric operators on an invariant domain of $\mathscr{H}$. Due to the translation invariance of the $W^{k}\left(x_{1}, \ldots, x_{k}\right)$ we have a strongly continuous unitary representation of the translation group on $\mathscr{H}$, with $\Omega$ as an invariant vector. From the analyticity properties of $G^{k}\left(x_{1}, \ldots, x_{k}\right)$ it follows that the infinitesimal generator of the time translations, $H$, is non negative, i.e. $H \geqq 0$. This canonical construction is such that

$$
W^{k}\left(x_{1}, \ldots, x_{k}\right)=\left(\Omega, \varphi\left(x_{1}\right) e^{i\left(t_{2}-t_{1}\right) H} \varphi\left(x_{2}\right) \ldots e^{i\left(t_{k}-t_{k}-1\right) H} \varphi\left(\boldsymbol{x}_{k}\right) \Omega\right) .
$$

Hence we have the following theorem:

Theorem 6.4. For real $\lambda$ and $|\lambda|<C^{-1} e^{-2 B-1}$ there is a Hilbert space $\mathscr{H}$ which carries a strongly continuous unitary representation of the translation group in space and time, with an invariant vector $\Omega$, and such that the polynomial algebra generated by $\varphi(f)$ with $f$ smooth $^{23}$ is represented by symmetric operators on an invariant domain of $\mathscr{H} . \Omega$ is cyclic with respect to the representation of the translation group and the algebra spanned by $\varphi(f)$; and $H$, the infinitesimal generator of the time translations, is non negative, $H \geqq 0$. Moreover, for $t_{1} \leqq \cdots \leqq t_{k}$ and $\left(x_{i}\right)_{0}=t_{i}$ $i=1, \ldots, k$ :

$$
G^{k}\left(x_{1}, \ldots, x_{k}\right)=\left(\Omega, \varphi\left(\boldsymbol{x}_{1}\right) e^{-\left(t_{2}-t_{1}\right) H} \ldots e^{-\left(t_{k}-t_{k-1}\right) H} \varphi\left(\boldsymbol{x}_{k}\right) \Omega\right) .
$$

Proof. All but the formula follows from what is said above. The formula follows from (6.6) and the fact that $W^{k}\left(x_{1}, \ldots, x_{k}\right)$ was taken to be the boundary values of $G^{k}\left(x_{1}, \ldots, x_{k}\right)$.

Theorem 6.5. For $\lambda$ real and $|\lambda|<C^{-1} e^{-2 B-1}, \Omega$ is the only translation invariant state of $\mathscr{H}$, and zero is a simple eigenvalue of $H$ with eigenvector $\Omega$.

${ }^{21}$ E.g. in $\mathscr{D}^{\prime}\left(\mathbb{R}^{n}\right), \mathscr{D}^{\prime}\left(\mathbb{R}^{n}\right)$ being the Schwartz' space of distributions over $\mathscr{D}\left(\mathbb{R}^{n}\right) \equiv C_{0}^{\infty}\left(\mathbb{R}^{n}\right)$. But the test function space can also be chosen to be more general, as can be seen from the preceding proofs.

22 See e.g. Ref. [16], Chapter 3, 3.4.

23 E.g. in Schwartz space $C_{0}^{\infty}(\mathbb{R})$ or $\mathscr{P}(\mathbb{R})$. 
Moreover the Wightman functions $W^{k}\left(x_{1}, \ldots, x_{k}\right)$ have also the cluster properties with respect to space translations, i.e. for $a=(0, \boldsymbol{a})$,

$$
W^{k+l}\left(x_{1}+a, \ldots, x_{k}+a, y_{1}, \ldots, y_{l}\right) \rightarrow W^{k}\left(x_{1}, \ldots, x_{k}\right) W^{l}\left(y_{1}, \ldots, y_{l}\right),
$$

in the sense of distributions ${ }^{21}$, as $|\boldsymbol{a}| \rightarrow \infty$.

Proof. From the formula of Theorem 6.4 and the cluster properties in the time directions of the $G$-functions as given in Theorem 5.2, we get that $\Omega$ is the only eigenvector with eigenvalue zero for $H$. The cluster properties of the Wightman functions are a direct consequence of the cluster properties of the $G$-functions in the space directions, since these imply that $U_{a}$ converges weakly to the projection on $\Omega$ as $|\boldsymbol{a}|$ tends to infinity, where $U_{a}$ is the unitary operator corresponding to the translation by $a=(0, a)$. This proves the theorem.

We shall now study the connection between the construction of the infinite volume Wightman functions, as we have done above, and the limit as $l \rightarrow \infty$ of the space cut-off vacuum state on elements of the algebra generated by all finite linear combinations of operators of the form $\alpha_{t_{1}}\left(e^{i s_{1} \varphi\left(f_{1}\right)}\right) \ldots \alpha_{t_{k}}\left(e^{i s_{k} \varphi\left(f_{k}\right)}\right), \alpha_{t}$ being the time automorphism given by Theorem 6.1.

As remarked in connection with (6.5), the quantities $\sigma_{l}^{k}\left(x_{1} s_{1}, \ldots, x_{k} s_{k}\right)$ converge, for real $\lambda$ with $|\lambda|<C^{-1} e^{-2 B-1}$ and for almost all $t_{i}=\left(x_{i}\right)_{0}$, as $l \rightarrow \infty$, to limit functions $\sigma^{k}\left(x_{1} s_{1}, \ldots, x_{k} s_{k}\right)$. Since $(-i \lambda)^{-k} \sigma_{l}^{k}\left(x_{1} s_{1}, \ldots, x_{k} s_{k}\right)$ satisfy the positive definiteness conditions, the limit functions $(-i \lambda)^{-k} \sigma^{k}\left(x_{1} s_{1}, \ldots, x_{k} s_{k}\right)$ satisfy also the positive definiteness conditions and can therefore be used in the same way as the Wightman functions to construct a representation space for the operators $e^{i s \varphi_{\varepsilon}(x)}$. From the construction it follows that $e^{i s \varphi_{\varepsilon}(x)}$ form a strongly continuous unitary group with infinitesimal generator $\varphi_{\varepsilon}(\boldsymbol{x})$. Using now (6.6) we get the identification of this representation with the one in Theorem 6.4. From this it follows by (6.5) that we have the following formula for the limit function $\sigma^{k}\left(x_{1} s_{1}, \ldots, x_{k} s_{k}\right)$ :

$$
\begin{aligned}
& \sigma^{k}\left(x_{1} s_{1}, \ldots, x_{k} s_{k}\right) \\
& \quad=(-i \lambda)^{k}\left(\Omega, e^{i s_{1} \varphi_{\varepsilon}\left(\boldsymbol{x}_{1}\right)} e^{-i\left(t_{2}-t_{1}\right) H} \ldots e^{-i\left(t_{k}-t_{k-1}\right) H} e^{i s_{k} \varphi_{\varepsilon}\left(\boldsymbol{x}_{k}\right)} \Omega\right)
\end{aligned}
$$

and hence correspondingly, for $t_{1} \leqq t_{2} \leqq \cdots \leqq t_{k}$ :

$$
\begin{aligned}
& \varrho^{k}\left(x_{1} s_{1}, \ldots, x_{k} s_{k}\right) \\
& \quad=(-\lambda)^{k}\left(\Omega, e^{i s_{1} \varphi_{\varepsilon}\left(x_{1}\right)} e^{-\left(t_{2}-t_{1}\right) H} \ldots e^{-\left(t_{k}-t_{k}-1\right) H} e^{i s_{k} \varphi_{\varepsilon}\left(x_{k}\right)} \Omega\right) .
\end{aligned}
$$


We have now, choosing $\chi_{\varepsilon}(x)$ positive definite:

Theorem 6.6. Let $\alpha_{t}$ be the time automorphism given by Theorem 6.1. For any real $\lambda$ with $|\lambda|<C^{-1} e^{-2 B-1}$ there exists a strongly dense linear subspace $\mathscr{W}$ of $\mathscr{H}_{n-\frac{1}{1}}^{-\frac{1}{1}}$ such that for all $f_{1}, \ldots, f_{k}$ in $\mathscr{W}$ and almost all $t_{i}$, $i=1, \ldots, k$ :

$$
\left(\Omega_{l}, \alpha_{t_{1}}\left(e^{i s_{1} \varphi\left(f_{1}\right)}\right) \ldots \alpha_{t_{k}}\left(e^{i s_{k} \varphi\left(f_{k}\right)}\right) \Omega_{l}\right)
$$

converges as $l \rightarrow \infty$ to

$$
\left(\Omega, \alpha_{t_{1}}\left(e^{i s_{1} \varphi\left(f_{1}\right)}\right) \ldots \alpha_{t_{k}}\left(e^{i s_{k} \varphi\left(f_{k}\right)}\right) \Omega\right),
$$

where $\Omega$ is the unique infinite volume vacuum given by the Theorems 6.4, 6.5. Moreover the limit is also equal, for almost all $t_{i}, i=1, \ldots, k$ to

$$
\left(\Omega, e^{i s_{1} \varphi\left(f_{1}\right)} e^{i\left(t_{2}-t_{1}\right) H} \ldots e^{i\left(t_{n}-t_{n-1}\right) H} e^{i s_{k} \varphi\left(f_{k}\right)} \Omega\right),
$$

where $H$ is the infinitesimal generator of the time translations given by Theorem 6.4.

Proof. By the definition of $\alpha_{l}^{l}$ and the fact that $\Omega_{l}$ is the eigenvector of $H_{l}$ to the eigenvalue $E_{l}$, we have

$$
\begin{aligned}
& \left(\Omega_{l}, \alpha_{t_{1}}^{l}\left(e^{i s_{1} \varphi_{\varepsilon}\left(\boldsymbol{x}_{1}\right)}\right) \ldots \alpha_{t_{k}}^{l}\left(e^{i s_{k} \varphi_{\varepsilon}\left(\boldsymbol{x}_{k}\right)}\right) \Omega_{l}\right) \\
& =\left(\Omega_{l}, e^{i s_{1} \varphi_{\varepsilon}\left(\boldsymbol{x}_{1}\right)} e^{-l\left(t_{2}-t_{1}\right) \bar{H}_{l}} \ldots e^{-i\left(t_{k}-t_{k-1}\right) \bar{H}_{l}} e^{i s_{k} \varphi_{\varepsilon}\left(\boldsymbol{x}_{k}\right)} \Omega_{l}\right),
\end{aligned}
$$

with $\bar{H}_{l}=H_{l}-E_{l}$.

By (6.5) this is equal to

$$
(-i \lambda)^{-k} \sigma_{l}^{k}\left(x_{1} s_{1}, \ldots, x_{k} s_{k}\right)
$$

and converges, as $l \rightarrow \infty$, to the limit functions

$$
(-i \lambda)^{-k} \sigma^{k}\left(x_{1} s_{1}, \ldots, x_{k} s_{k}\right),
$$

for all real $\lambda$ with $|\lambda|<C^{-1} e^{-2 B-1}$ and almost all $t_{i}$. By (6.7) these limit functions are equal to

$$
\left(\Omega, e^{i s_{1} \varphi_{\ell}\left(\boldsymbol{x}_{1}\right)} e^{-i\left(t_{2}-t_{1}\right) H} \ldots e^{-l\left(t_{k}-t_{k}-1\right) H} e^{\imath s_{k} \varphi_{\varepsilon}\left(\boldsymbol{x}_{k}\right)} \Omega\right) .
$$

We have therefore

$$
\begin{aligned}
& \lim _{l \rightarrow \infty}\left(\Omega_{l}, \alpha_{t_{1}}^{l}\left(e^{l S_{1} \varphi_{\varepsilon}\left(\boldsymbol{x}_{1}\right)}\right) \ldots \alpha_{t_{k}}^{l}\left(e^{l S_{k} \varphi_{\varepsilon}\left(\boldsymbol{x}_{k}\right)}\right) \Omega_{l}\right) \\
& =\left(\Omega, e^{i S_{1} \varphi_{\varepsilon}\left(\boldsymbol{x}_{1}\right)} e^{-i\left(t_{2}-t_{1}\right) H} \ldots e^{-i\left(t_{k}-t_{k}-1\right) H} e^{l S_{k} \varphi_{\varepsilon}\left(\boldsymbol{x}_{k}\right)} \Omega\right) .
\end{aligned}
$$


Introduce now the functions $f_{i}(\boldsymbol{y})$ on $\mathbb{R}^{n-1}$, defined, for each $\boldsymbol{x}_{i} \in \mathbb{R}^{n-1}$ by: $f_{l}(\boldsymbol{y})=\chi_{\varepsilon}\left(\boldsymbol{x}_{i}-\boldsymbol{y}\right)$. These functions belong to $\mathscr{H}_{n-1}^{-\frac{1}{2}}$ and one has

$$
\varphi_{\varepsilon}\left(\boldsymbol{x}_{i}\right)=\int \varphi(\boldsymbol{y}) f_{i}(\boldsymbol{y}) d \boldsymbol{y}=\varphi\left(f_{i}\right)
$$

Introducing these identities in (6.9) we obtain:

$$
\begin{aligned}
& \lim _{l \rightarrow \infty}\left(\Omega_{l}, \alpha_{t_{1}}^{l}\left(e^{i s_{1} \varphi\left(f_{1}\right)}\right) \ldots \alpha_{t_{k}}^{l}\left(e^{l s_{k} \varphi\left(f_{k}\right)}\right) \Omega_{l}\right) \\
& =\left(\Omega, e^{i s_{1} \varphi\left(f_{1}\right)} e^{-i\left(t_{2}-t_{1}\right) H} \ldots e^{-l\left(t_{k}-t_{k}-1\right) H} e^{i s_{k} \varphi\left(f_{k}\right)} \Omega\right) .
\end{aligned}
$$

On the other hand, because of the strong convergence on $\mathscr{A}_{0}$ of $\alpha_{t}^{l}$, given by Theorem 6.1 , and because of the uniform bound $\left\|\Omega_{l}\right\|=1$, we have that

$$
\begin{aligned}
& \lim _{l \rightarrow \infty}\left(\Omega_{l}, \alpha_{t_{1}}^{l}\left(e^{i s_{1} \varphi\left(f_{1}\right)}\right) \ldots \alpha_{t_{k}}^{l}\left(e^{l s_{k} \varphi\left(f_{k}\right)}\right) \Omega_{l}\right) \\
= & \lim _{l \rightarrow \infty}\left(\Omega_{l}, \alpha_{t_{1}}\left(e^{i s_{1} \varphi\left(f_{1}\right)}\right) \ldots \alpha_{t_{k}}\left(e^{i s_{k} \varphi\left(f_{k}\right)}\right) \Omega_{l}\right) .
\end{aligned}
$$

This together with (6.10) are the formulae of the theorem, which are therefore proven for $f_{i}(\boldsymbol{y})=\chi_{\varepsilon}\left(\boldsymbol{x}_{i}-\boldsymbol{y}\right), i=1, \ldots, k$. The rest follows from the fact that the set $\mathscr{W}$ of all finite real linear combinations of these functions $f_{i}$, for all $\boldsymbol{x}_{i} \in \mathbb{R}^{n-1}, i=1, \ldots, k$ and all positive integers $k$ is dense in $\mathscr{H}_{n-1}^{-\frac{1}{2}}$, since the $f_{i}$ run over the set of all translates of the function $\chi_{\varepsilon}(-\boldsymbol{y})=\chi_{\varepsilon}(\boldsymbol{y})$ for which $\tilde{\chi}_{\varepsilon}(\boldsymbol{p})(\mu(\boldsymbol{p}))^{-\frac{1}{2}}>0$ for almost every $\boldsymbol{p} \in \mathbb{R}^{n-1}$, $\tilde{\chi}_{\varepsilon}(\boldsymbol{p})$ being the Fouriertransform of the symmetric, positive definite function $\chi_{\varepsilon}(\boldsymbol{y})$.

Remark. Theorem 6.6 connects the limit of the space cut-off vacuum state on an algebra defined in terms of the time automorphism constructed by Streater and Wilde [6d] with the infinite volume quantities we have constructed in Theorems 6.2 to 6.5 .

Remark. Since, by Theorem 5.4, for $|\lambda|<C^{-1} e^{-2 B-1}$ one has that the vacuum energy density $\left|B_{l}\right|^{-1} E_{l}$ converges to $\tilde{\varepsilon}$ as $l \rightarrow \infty$ and moreover the interaction $V_{l}$ is bounded in norm by a constant limes $\left|B_{l}\right|$, we obtain the estimate

$$
\left|\left(\Omega_{l}, H_{0} \Omega_{l}\right)\right| \leqq C_{1}\left|B_{l}\right|
$$

where $C_{1}$ is independent of $l$.

This inequality and the fact that the Wightman functions tend, as $l \rightarrow \infty$, to the translation invariant Wightman functions could be used to prove, along the lines of [17], that the representation space in the infinite volume limit is locally Fock. 
Acknowledgements. We are very grateful to Prof. Dr. R. Streater and Dr. I. F. Wilde for helpful correspondence and corrections concerning an earlier version of Section 6 . It is a pleasure for the first named author (S.A.) to thank the Mathematics Institute of Oslo University for the hospitality.

\section{References}

1. Glimm,J., Jaffe, A.: Quantum Field Theory Models, in Statistical Mechanics and Quantum Field Theory. Les Houches Summer School, 1970, Ed. by De Witt and Stora. New York: Gordon \& Breach 1971. Also: Glimm, J., Jaffe, A.: Boson Quantum Field Models, 1971. London Lectures, Ed. by R. Streater (to appear).

2. Glimm, J., Jaffe, A.: The $\lambda \Phi_{2}^{4}$ Quantum Field Theory without Cutoffs IV. Perturbation of the Hamiltonian, Preprint 1972.

3. Streater, R.: On the connection between spectrum condition and Lorentz invariance. Commun. math. Phys. 26, 109-120 (1972).

4. Høegh-Krohn, R.: A general class of quantum fields without cut-offs in two spacetime dimensions. Commun. math. Phys. 21, 244-251 (1971).

5. Schrader, R.: Yukawa quantum field theory in two space-time dimensions without cut-offs. Annals of Physics 70, 412 - 457 (1972).

6. a) Høegh-Krohn, R.: Boson fields under a general class of cut-off interactions. Commun. math. Phys. 12, 216-225 (1969).

b) Høegh-Krohn, R.: Boson fields under a general class of local relativistic invariant interactions. Commun. math. Phys. 14, 171-184 (1969).

c) Høegh-Krohn, R.: Boson fields with bounded interaction densities. Commun. math. Phys. 17, 179-193 (1970).

d) Streater, R.F., Wilde,I.F.: The time evolution of quantized fields with bounded quasi-local interaction density. Commun. math. Phys. 17, 21-32 (1970).

7. a) Efimov, G.V.: Essentially non linear interaction Lagrangians and non localized quantum field theory. Theor. and Mathem. Phys. 2, 26-40 (1970).

b) Ya Petrina,D., Skripnik, V.I.: Kirkwood-Salzburg equations for the coefficient functions of the scattering matrix. Theor. and Mathem. Phys. 8, 896-903 (1971).

c) Fivel, D.: Construction of unitary, covariant $S$ matrices defined by convergent perturbation series. Phys. Rev. D, 4, 1653-1662 (1971).

8. Symanzik, K.: Lectures on euclidean quantum field theory, in Local Quantum Theory, International School of Physics "Enrico Fermi", Varenna, Ed. by R. Jost. New York: Academic Press 1969.

9. a) Nelson,E.: Quantum fields and Markov fields. Proc. 1971 AMS Summer Conference.

b) Nelson,E.: The free Markov field. Princeton University Preprint, Mathematics Department, 1972.

c) Nelson,E.: Construction of quantum fields from Markoff fields. Princeton University Preprint, Math. Deptm., 1972.

10. Guerra,F.: Uniqueness of the vacuum energy density and Van Hove phenomenon in the infinite volume limit for two-dimensional self-coupled Bose fields. Phys. Rev. Lett. 28, 1213-1215 (1972).

11. Guerra, F., Rosen, L., Simon, B.: Nelson's symmetry and the infinite volume behaviour of the vacuum in $P(\Phi)_{2}$, Commun. math. Phys. 27, 10-22 (1972).

12. Gelfand, I. M., Ya Vilenkin, N.: Generalized Functions, Vol. 4. New York: Academic Press 1964.

13. a) Segal, I.: Tensor algebras over Hilbert spaces I. Trans. Am. Mathem. Soc. 81, $106-134$ (1956).

b) Simon, B., Høegh-Krohn, R.: Hypercontractive semigroups and two-dimensional self-coupled Bose fields. J. Funct. Analys. 9, 121-180 (1972). 
14. Høegh-Krohn, R.: Infinite dimensional analysis with applications to self-interacting boson fields in two space time dimensions. Talk given at Aarhus Conference on Functional Analysis, Spring 1972.

15. Ruelle, D.: Statistical Mechanics, Rigorous Results. New York: W. A. Benjamin, Inc. 1969. Especially ch. 4.

16. Streater,R.F., Wightman, A.: PCT, Spin \& Statistics, and all that. New York: W A Benjamin 1964.

17. Glimm,J., Jaffe, A.: The $\lambda\left(\Phi^{4}\right)_{2}$ quantum field theory without cut-offs. III. The physical vacuum. Acta Mathem. 125, 203-267 (1970).

Sergio Albeverio

R. Høegh-Krohn

Institute of Mathematics

University of Oslo

Blindern, Oslo, Norwegen 